\title{
On spaces of holomorphic functions
}

by

\section{S. ROLEWICZ (Warszawa)}

In this paper we consider locally convex metrisable spaces whose elements are analytic functions of several variables. We develop Kolmogorov's ideas [14], which concern the existence of continuous linear mappings at such spaces. In particular, we consider the following problem: For which domains $D_{1}$ and $D_{2}$ in Euclidean complex spaces of dimensions $k_{1}$ and $k_{2}$, respectively, the spaces $\mathscr{H}(D)$ and $\mathscr{H}\left(D_{1}\right)$ - of all holomorphic (1) functions defined on these domains - are isomorphic (linearly homeomorphic).

Our results (Corollaries 4.1, 4.2, and 4.3) contain as particular cases a result of Kolmogorov which states that if $D_{1}$ and $D_{2}$ are polyeylinders and $\operatorname{dim} D_{1} \neq \operatorname{dim} D_{2}$, then the spaces $\mathscr{H}\left(D_{1}\right)$ and $\mathscr{H}\left(D_{2}\right)$ are not isomorphie, and a result of Pełezyński [21] stating that the spaces of all entire functions of one variable and $\mathscr{H}\left(C_{0}\right)-$ of all holomorphic functions defined for $|z|<1$ - are not isomorphic.

We also consider the spaces of all holomorphic functions having a given degree of growth.

Similarly to Kolmogorov [14] and Pelczyński [21], in order to establish that given spaces are not isomorphic we compute the so-called "approximative dimension", which is an invariant of linear homeomorphisms. Our method of computing approximative dimension is different from that of Kolmogorov-Tichomirov [15] and Erochin [9]. It is based on finding some matrix representations of a given space by basic expansions. A matrix representation determines the isomorphic structure of the space, in particular it determines its approximative dimension. Matrix representatiens of some spaces of holomorphic functions are found in $\S 3$ and in $\S 5$, section 2 . Examples given in $\S 5$ show that non-isomorphic spaces can have the same approximative dimension. However, under the assumption that spaces have matrix representations of a special form, the equality of approximative dimensions implies the isomorphism of the spaces (Theorem 5.3).

(1) i. e. analytic and one-valued functions. 
In many cases the matrix representation is obtained by replacing every function of a given space by its Taylor coefficients. This allows us to find estimations of Taylor coefficients of holomorphic functions for some functional classes. Examples of such estimations are given in $\S 6$.

Most of the results of this paper were announced in [23] and [24]. Recently some results have been obtained independently by Aizenberg and Mitiagin [1], [2].

The results of $\S 1$, section 3 , and $\S 5$ were obtained in collaboration with C. Bessaga and A. Pelezyński.

The author would like to express his warmest thanks to Dr C. Bessaga for his help in the preparation of this paper.

\section{\$ 1. Preliminaries}

1. $B_{0}$-spaces; bases. In the sequel we shall consider only $B_{0}$-spaces, i. e. locally convex complete metric linear spaces. For the basic properties of such spaces see Mazur and Orlicz [18] or Bourbaki's monograph $[6]$, in which they are called $F$-spaces. Now we recall only that the topology in a $B_{0}$-space $X$ can be given by a non-decreasing sequence of pseudonorms $\left(\|\cdot\|_{a}\right), \alpha=1,2, \ldots$

Linear topological spaces $X$ and $Y$ are said to be isomorphio (written $X \approx Y$ ) if and only if there exists a linear homeomorphic mapping from $X$ onto $Y$. The symbol $X \neq Y$ will denote that the spaces $X$ and $Y$ are not isomorphic.

Let $\left(X_{j}\right)$ be a sequence of $B_{0}$-spaces whose topologies are given by sequences of pseudonorms $\left(\|\cdot\|_{a j}\right), j=1,2, \ldots$, respectively. The space $\mathscr{X}$ whose elements are sequences $\left(x_{j}\right), x_{j}$ being in $X_{j}$, with the topology determined by the pseudonorms $\left\|\left(x_{j}\right)\right\|_{\alpha}=\sup \left\{\left\|x_{i}\right\|_{\beta i}: i, \beta \leqslant \alpha\right\}$ will be called a product in sense $s$ of spaces $X_{j}$ and denoted by the symbol $\left(X_{1} \times X_{2} \times \ldots\right)_{s}$.

A series $\sum_{n=1}^{\infty} x_{n}$ of elements of a $B_{0}$-space $X$ is said to be absolutely convergent if and only if $\sum_{n=1}^{\infty}\left\|x_{n}\right\|<\infty$ for every continuous pseudonorm $\|\cdot\|$ defined on $X$.

A sequence $\left(e_{n}\right)$ with $e_{n} \in X$ is called a basis (an absolute basis) of the space $X$ if and only if every vector $x$ in $X$ may be uniquely represented as the sum of a convergent (absolute convergent) series:

$$
x=\sum_{n=1}^{\infty} t_{n} e_{n} \quad\left(t_{n} \text {-sealars }\right) .
$$

2. Köthe spaces. We shall ecnsider a class of "Stufenräume" intrcducc d by Kc̈the. Let $\mathscr{N}^{k} d \in$ ncte the set of all systems of $k$ non-negative integers. Suppose we are given a $(k+1)$-dimensional matrix of num- bers $a_{a_{n}}$, where $\alpha=1,2, \ldots, \boldsymbol{n} \epsilon \mathscr{N}^{k}$, such that

(11) $\quad a_{a_{n}} \geqslant 0, \quad \sup a_{a_{n}}>0, \quad a_{a n} \leqslant a_{\beta n}$ for $\alpha \leqslant \beta$.

A Köthe space $\mathscr{M}\left(c_{a_{n}}\right)$ is the space of all (k-fold) sequences $\xi=\left(\xi_{n}\right)$ of complex numbers such that

$$
\|\xi\|_{\alpha}=\sup _{\boldsymbol{n}} a_{a n}\left|\xi_{n}\right|<\infty \quad(\alpha=1,2, \ldots) .
$$

Addition and multiplication by scalars in this space is defined as usual; the topology is given by pseudonorms $\|\cdot\|_{\alpha}$.

In the case of $k=1$ we shall write $n$ instead of $n$; spaces $\mathscr{M}\left(a_{a n}\right)$ will be called single Köthe spaces.

If $X=\mathscr{M}\left(a_{a_{n}}\right)$, we shall say that $X$ has a matrix representation $\mathscr{M}\left(a_{a n}\right)$.

THEOREM 1.1 [22]. Let $X$ be a $B_{0}$-space with topology determined by a non-decreasing sequence of pseudonorms (\|. $\left.\|_{a}\right)$ and with a basis $\left(e_{n}\right)$. Let $a_{\alpha n}=\left\|e_{n}\right\|_{\alpha}$. If

(12) for every a there exists a $\beta$ such that $\sum_{n=1}^{\infty} a_{a n} / a_{\beta n}<\infty$ (here we under$\operatorname{stand} 0 / 0=0)$,

then $x=\mathscr{M}\left(a_{a n}\right)$.

For the proof see [4].

A $B_{0}$-space $X$ with a basis satisfying condition (12) is said to be nuclear $\left({ }^{2}\right)$.

Remark 1.1. It follows immediately from Theorem 1.1 that if a set of vectors $\left(e_{n}\right)$ can be reordered in such a way as to constitute a basis of $X$ and if for every $\alpha$ there is a $\beta$ such that $\sum_{n \in \mathcal{K}^{k}}\left\|e_{\boldsymbol{n}}\right\| \alpha /\left\|e_{\boldsymbol{n}}\right\|_{\beta}<\infty$, then $X \approx \mathscr{M}\left(\left\|e_{n}\right\|_{\alpha}\right)$.

3. Approximative dimension. Let $A, B$ be subsets of a linear space $X$. By $M(A, B, \varepsilon)$ we shall denote the maximal number $n$ of such points $x_{1}, \ldots, x_{n}$ in $A$ that $x_{i}-x_{j} \xi 2 \varepsilon B$ for $i \neq j . M(A, B)$ will denote the class of all non-negative functions $\varphi(\varepsilon)$ defined for $\varepsilon>0$ such that for sufficiently small $\varepsilon$

$$
\varphi(\varepsilon) \geqslant M(A, B, \varepsilon)
$$

Now let us suppose that $X$ is a linear topological space. Let $\mathscr{U}$ be the class of all open sets in $X$ and $\mathfrak{S}$ - the class of all bounded sets in this space. The class of functions:

$$
\Phi(X)=\bigcap_{U \in \mathfrak{Q}} \bigcap_{B \in \mathfrak{E}} M(B, U)
$$

is called the approximative dimension of space $X$ (Kolmogorov [14]).

(2) For general definition of nuclear spaces see [11]. 
Pelczyński's considerations [21] lead to the distinction of another class, namely:

$$
\bigcap_{\nabla \in \mathfrak{A}} \bigcup_{U \in \mathfrak{A}} M(U, V)
$$

In the case of $B_{0}$-spaces both classes are identical [22].

We shall say that the approximative dimension of space $X$ is equal to, less than or equal to, and less than that of $Y$ (written $\mathrm{d}_{a} X=\mathrm{d}_{a} Y$, $\left.\mathrm{d}_{a} X \leqslant \mathrm{~d}_{a} Y, \quad \mathrm{~d}_{a} Y<\mathrm{d}_{a} Y\right) \quad$ if $\Phi(X)=\Phi(Y), \quad \Phi(X) \supset \Phi(Y), \quad$ and $\Phi(X) \supsetneqq \Phi(Y)$, respectively.

It is easily seen that the approximative dimension is an isomorphio invariant of spaces; moreover if $Y$ is a subspace or a linear (continuous) image of $X$, then $\mathrm{d}_{a} Y \leqslant \mathrm{~d}_{a} X$.

In the case of $B_{0}$-spaces it is more convenient to deal with formula (13): instead of the family $\mathfrak{U}$ one may take an arbitrary basis $\left(U_{n}\right)$ of neighbourhoods of zero.

Let $X=M\left(a_{a n}\right)$, with $n \in \mathscr{N}^{k}$, be a nuclear Köthe space and let

$$
M_{\alpha \beta}(X ; \varepsilon)=\prod_{\boldsymbol{n} \in \mathscr{S}^{k}} \mathrm{E}\left(1+a_{\alpha_{\boldsymbol{n}}} /\left(\varepsilon a_{\beta \boldsymbol{n}}\right)\right)\left(^{3}\right) .
$$

Denote by $M_{a \beta}(X)$ the class of all non-negative functions $\varphi(\varepsilon)$ defined for $\varepsilon>0$ such that $\varphi(\varepsilon) \geqslant M_{\alpha \beta}(\varepsilon)$ for sufficiently small $\varepsilon$.

THEOREM 1.2. If $X=\mathscr{M}\left(a_{\alpha, n}\right)$, with $\boldsymbol{n} \in \mathscr{N}^{k}$, is a nuolear Köthe space, then $\Phi(X)=\bigcap_{a} \bigcup_{\beta} M_{\alpha \beta}(X)\left(^{4}\right)$.

THEOREM $1.3\left(^{(5)}\right.$. Let matrices $a_{a n}^{(j)}(j=1, \ldots, k)$ satisfy condition (12) and let

$$
a_{a n}=a_{a n_{1}}^{(1)} \cdot \ldots \cdot a_{a n_{k}}^{(k)} ; \quad N_{a \beta j}(\varepsilon)=\overline{\overline{\left\{n: a_{\beta n}^{(j)} / \overline{a_{a n}^{(j)} \geqslant \varepsilon}\right.}}\left({ }^{6}\right)
$$

\section{Then}

$$
\frac{1}{2} \log \frac{1}{\varepsilon} \prod_{j=1}^{k} N_{a \beta j}(\sqrt[2 k]{\varepsilon}) \leqslant \log M_{\alpha \beta}\left(\mathscr{M}\left(a_{a n}\right) ; \varepsilon\right) \leqslant \log \left(1+\frac{1}{\varepsilon}\right) \prod_{j=1}^{k} N_{a \beta j}(\varepsilon) .
$$

Proof. Write

$$
P_{\alpha \beta}(\varepsilon)=\left\{n: a_{\alpha n}^{(1)} / a_{\beta n}^{(1)} \geqslant \frac{1}{\varepsilon}\right\} \times \ldots \times\left\{n: a_{\alpha n}^{(k)} / a_{\beta n}^{(k)} \geqslant \frac{1}{\varepsilon}\right\} .
$$

Right-hand inequality. We have

(3) $\mathrm{E} a$ denotes the greatest integer in $a$.

(4) Proofs of theorems 1.2, 1.4, and 1.5 will be published in Studia Math. in a joint paper with C. Bessaga and A. Pełczyński.

(5) ef. Mitiagin [19], theorem 1.

(6) The symbol $\overline{\bar{A}}$ denotes the cardinality (number of elements) of the set $A$.

$$
\prod_{n \in \mathcal{H}^{k}} \mathrm{E}\left(1+a_{a_{n}} /\left(\varepsilon a_{\beta \boldsymbol{n}}\right)\right)=\prod_{\boldsymbol{n} \in P_{\alpha \beta}(\varepsilon)} \mathrm{E}\left(1+a_{a_{n}} /\left(\varepsilon a_{\beta \boldsymbol{n}}\right)\right) \leqslant\left(1+\frac{1}{\varepsilon}\right)^{\overline{\overline{P_{\alpha \beta}(\varepsilon)}}} .
$$

Since $\overline{\overline{P_{\alpha \beta}(\varepsilon)}}=\prod_{j=1}^{k} N_{a \beta j}(\varepsilon)$, we obtain the required inequality.

Left-hand inequality. Since $\mathrm{E}\left(1+a_{a \boldsymbol{n}} /\left(\varepsilon a_{\beta_{\boldsymbol{\beta}}}\right)\right) \geqslant 1 / \sqrt{\varepsilon}$ for $n \in P_{a \beta}(\sqrt[2 k]{\sqrt{\varepsilon})}$, we have

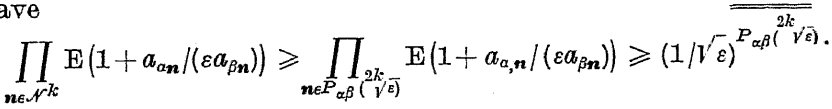

THEOREM 1.4. Let matrices $\left(a_{a p}\right),\left(b_{a q}\right)$ satisfy condition (12), where $\boldsymbol{p}=\left(n_{1}, \ldots, n_{r}\right) \in \mathscr{N}^{r}, \boldsymbol{q}=\left(n_{r+1}, \ldots, n_{k}\right) \in \mathscr{N}^{k-r}$, and let $c_{a n}=a_{a \boldsymbol{p}} \cdot b_{a \boldsymbol{\alpha}}$, where $\boldsymbol{n}=\left(n_{1}, \ldots, n_{k}\right) \in \mathscr{N}^{k}$. Then

$$
M_{a \beta}\left(\mathscr{M}\left(o_{a \boldsymbol{n}}\right) ; \varepsilon\right)=\prod_{\boldsymbol{q} \epsilon=-r_{k-r}} M_{a \hat{p}}\left(\mathscr{M}\left(a_{a \boldsymbol{p}}\right) ; \varepsilon b_{\beta \boldsymbol{q}} / b_{a \boldsymbol{q}}\right) .
$$

THEOREM 1.5. Let $X_{n}$ be a sequence of $B_{0}$-spaces. If $\mathrm{d}_{a}\left(X_{i} \times X_{j}\right)=$ $=\mathrm{d}_{a} X_{1}$, then $\mathrm{d}_{a}\left(X_{1} \times X_{2} \times \ldots\right)_{s}=\mathrm{d}_{a} X_{1}$. In particular, if $\mathrm{d}_{a} X=$ $=\mathrm{d}_{a}(X \times X)$, then $\mathrm{d}_{a} X=\mathrm{d}_{a}(X \bar{\otimes} s)$. (Symbol $X \times Y$ denotes the Cartesian product of spaces $X$ and $Y$ ).

4. Spaces $\mathscr{H}_{\mu}(D)$. Let $D$ be a domain in a $k$-dimensional Euclidean complex space. Let $\mu(\varepsilon, z)$ be a non-negative function defined for $0<\varepsilon<1$, $z \in D$, non-increasing with respect to $\varepsilon$ and $\operatorname{such}$ that $\lim \mu(\varepsilon, z)>0$, for every $z$ in $D$. By $\mathscr{H}_{\mu}(D)$ we shall denote the space of all holomorphic functions $x=x(z)$ defined on $D$ and such that

$$
\left.\|x\|_{\varepsilon}=\sup _{z \in D} \mid x(z)\right] \mu(\varepsilon, z)<+\infty
$$

with the topology induced by the pseudonorms $\|\cdot\|_{\varepsilon}$.

Since $\mu(\varepsilon, z)$ is non-increasing with respect to $\varepsilon$, the topology of the space $\mathscr{H}_{\mu}(D)$ may be given by the sequence of pseudonorms $\|\cdot\|_{1 / n}$. Hence $\mathscr{H}_{\mu}(D)$ is a $B_{0}$-space.

If $A_{\varepsilon}(0<\varepsilon<1)$ is a non-increasing family of compact sets such that $\bigcup_{\varepsilon} A_{\varepsilon}=D$ and $\mu(\varepsilon, z)=\chi_{A}(z)$ (the characteristic function of the set $A)^{\varepsilon}$, then $\mathscr{H}_{\mu}(D)$ is the space of all holomorphic functions defined on $D$ with the topology of almost uniform convergence. This space will be briefly denoted by $\mathscr{H}(D)$.

If $D$ is the whole $k$-dimensional Euclidean complex space, then instead of $\mathscr{H}_{\mu}(D)$ we shall write $\mathscr{H}_{\mu}$.

The symbols $C$ and $C_{0}$ will be reserved further for denoting the whole complex plane and the interior of the unit circle; $\bar{C}$ will denote 
the extended complex plane, i. e. $\bar{C}=C+\{\infty\} . O^{r}$ (resp. $C_{0}^{r}$ ) will denote the Cartesian product of $r$ copies of $C$ (resp. $C_{0}$ ).

THEOREM 1.6. Suppose we are given a space $\mathscr{H}_{\mu}=\mathscr{H}_{\mu}\left(C^{k}\right)$ such that the function $\mu(\varepsilon, z)$ depends only on $\left|z_{1}\right|, \ldots,\left|z_{l_{i}}\right|$, and let $z^{\mathbf{n}}=z_{1}^{n_{1}} \ldots$ $\ldots z_{k}^{n_{k}}$, for $\boldsymbol{n} \in \mathscr{M}^{k}$. If for every $0<\varepsilon<1$ there exists an $\varepsilon^{\prime}$ such that

$$
\sum_{\boldsymbol{n} \in \mathcal{H}^{k}}\left|z^{\boldsymbol{n}-1}\right|_{\varepsilon} /\left|z^{\mathbf{n}}\right|_{\varepsilon^{\prime}}<\infty
$$

then the mononomials $z^{\mathbf{n}}$ constitute a basis of $\mathscr{H}_{\mu}$. The space $\mathscr{H}_{\mu}$ has a matrix representation $M\left(\left|z^{n}\right|_{\varepsilon_{a}}\right)$, where $\left(\varepsilon_{a}\right)$ is an arbitrary sequence such that $0<\varepsilon_{\alpha}<1, \lim _{\alpha \rightarrow \infty} \varepsilon_{a}=0$.

Pro of. Every entire function, in particular every function from $\mathscr{H}_{\mu}$, can be uniquely represented as the sum of the series

$$
x(z)=\sum_{\boldsymbol{n} \in \mathcal{H}^{\prime k}} c_{\boldsymbol{n}} z^{\boldsymbol{n}}
$$

which is absolutely and almost uniformly convergent ([10], p. 74). By Cauchy formula $\left([10]\right.$, p. 24) we obtain $\left|e_{n-1}\right| \leqslant\left(1 / r^{\boldsymbol{n}}\right) \max _{\left|z_{i}\right|=v_{i}}|x(z)|$, where $r^{\boldsymbol{n}}=r_{1}^{n_{1}} \ldots r_{k}^{n_{k}}$, for every $\boldsymbol{n}$ in $\mathscr{N}^{k}$. Therefore

Hence

$$
\left|c_{n-1} z^{\boldsymbol{n}}\right| \leqslant \max \left\{|x(\xi)|: \xi=\left(\xi_{1}, \ldots, \xi_{k}\right),\left|\xi_{i}\right|=\left|z_{i}\right|\right\} .
$$

$$
\mu(\varepsilon, z)\left|\boldsymbol{c}_{\boldsymbol{n}-1} z^{\boldsymbol{n}}\right| \leqslant \mu(\varepsilon, z) \max _{\left|\xi_{i}\right|=\left|z_{i}\right|}\left|x\left(\xi_{i}\right)\right| .
$$

Now, the assumption that $\mu(\varepsilon, z)$ depends on $\left|z_{1}\right|, \ldots,\left|z_{z_{c}}\right|$ gives us

$$
\left\|c_{n-1} z^{n}\right\|_{\varepsilon} \leqslant\|x\|_{\varepsilon} \quad \text { for every } \quad n \in \mathscr{N}^{k} .
$$

From this inequality we deduce by (15) that the series (1.6) is convergent in the topology of the space $\mathscr{H}_{\mu}$. Since, by (14), convergence in $\mathscr{H}_{\mu}$ implies uniform convergence, expansion (16) is unique also in the topology of $\mathscr{H}_{\mu}$, i. e. $\left(z^{n}\right)$ is a basis of $\mathscr{H}_{\mu}$.

The matrix representation of $\mathscr{H}_{\mu}$ follows from formula (15) and Theorem 1.1.

5. Tensor products. Let $X$ and $Y$ be $B_{0}$-spaces, $X^{*}$ and $X^{*}-$ their conjugate spaces. The space of all bilinear forms defined on the Cartesian product $X^{*} \times Y^{*}$ is called the tensor product of spaces $X$ and $Y$ (written $X \hat{\hat{\otimes}} Y$ ) [11].

Further we shall make use of the following three facts concerning tensor products:

(17) If $X_{1} \approx X, \quad Y_{1} \approx Y$, then $X \hat{\hat{\otimes}} Y \approx X_{1} \hat{\hat{\otimes}} Y_{1}, \quad X \hat{\hat{\otimes}}(X \times Z) \approx$ $\approx(X \hat{\hat{\otimes}} \bar{Y}) \times(X \hat{\hat{\otimes}} Z)$;
(18) If $D_{1}$ and $D_{2}$ are open sets in $C^{k}$ and $C^{k_{1}}$ respectively, then $\mathscr{H}\left(D_{1} \times D_{2}\right) \approx$ $\approx \mathscr{H}\left(D_{1}\right) \hat{\hat{\otimes}} \mathscr{H}\left(D_{2}\right)\left({ }^{7}\right)$;

(19) If $X \approx M\left(a_{a p}\right), \quad \bar{Y} \approx M\left(b_{a q}\right)$, with $\boldsymbol{p}=\left(n_{1}, \ldots, n_{r}\right) \in \mathscr{N}^{r}, \quad \boldsymbol{q}=$ $=\left(n_{r+1}, \ldots, n_{k}\right) \in \mathcal{S}^{k-r}$, are nuclear Köthe spaces and $c_{a n}=a_{a p} b_{a \boldsymbol{q}}$ for $\boldsymbol{n}=(\boldsymbol{p}, \boldsymbol{q})=\left(n_{1}, \ldots, n_{k}\right) \in \mathscr{W}^{k}$, then $X \hat{\mathrm{\Theta}} Y \approx \mathscr{M}\left(c_{\text {an }}\right)$.

§ 2. Some isomorphic relations between spaces $\mathscr{H}(D)$

LEMMA 2.1. Suppose we are given an analytic transformation of an open plane set $D_{1}$ onto an open plane set $D_{2}$. Then the space $\mathscr{H}\left(D_{2}\right)$ is isomorphic to a subspace of $\mathscr{H}\left(D_{1}\right)$.

Proof. Let

$$
(T x)(z)=x(\varphi(z)) .
$$

We shall show that $T$ is an isomorphism mapping of $\mathscr{H}\left(D_{2}\right)$ into $\mathscr{H}\left(D_{1}\right)$. Obviously $T$ is a linear one-to-one mapping. To show that it is continuous let us choose $y_{n} \in T\left(\mathscr{H}\left(D_{2}\right)\right)(n=1,2, \ldots)$ such that $y_{n} \rightarrow 0$. Let $x_{n}=T y_{n} \quad(n=1,2, \ldots)$. Suppose that $x_{n} \rightarrow 0$. Then there is a compact set $K_{2} \subset D_{2}$ such that $\varlimsup_{n}\left\|x_{n}\right\|_{K_{2}}=\varlimsup_{n_{n} \sup _{2}}\left|x_{n}(w)\right|>0$. Since for every compact $K_{2} \subset D_{2}$ there is a compact set $K_{1} \subset D_{1}$ such that $\Phi\left(K_{1}\right) \supset K_{2}$, we have $\overline{\lim }\left\|y_{n}\right\|_{K_{1}} \geqslant \varlimsup \operatorname{sim} \sup \left|x_{n}(w)\right| \geqslant \varlimsup\left\|x_{n}\right\|_{K_{2}}>0$, which leads to a contradiction.

$$
w_{6} \Phi^{-1}\left(K_{1}\right)
$$

Lencs 2.2. Let $D$ be an arbitrary k-dimensional domain. Then the space $\mathscr{H}(C \times D)$ (resp. the space $\left.\mathscr{H}\left(C_{0} \times D\right)\right)$ is isomorphic to its cartesian square.

Proof. First we note that the space $\mathscr{H}(C)$ is isomorphic to its Cartesian square. Let us put $h x=\left(x_{1}, x_{2}\right)$, where

$$
x_{1}(z)=\frac{x(z)+x(-z)}{2}, \quad x_{2}(z)=\frac{x(z)-x(-z)}{2 z},
$$

for any $x \in \mathscr{H}(C)$. It is easily seen that $h$ is the required isomorphism. In the general case, according to (17) and (18), we have

$$
\begin{aligned}
\mathscr{H}(C \times D) & \approx \mathscr{H}(C) \otimes \mathscr{H}(D)=(\mathscr{H}(C) \times \mathscr{H}(C)) \otimes \mathscr{H}(D) \\
& \approx(\mathscr{H}(C) \otimes \mathscr{H}(D)) \times \mathscr{H}(C) \otimes \mathscr{H}(D) \approx \mathscr{H}(C \otimes D) \times \mathscr{H}(C \otimes D) .
\end{aligned}
$$

The proof for the space $\mathscr{H}\left(C_{0} \times D\right)$ is analogous.

( ${ }^{7}$ This formula follows from the fact that every function $x(z)$ in $\mathscr{H}\left(D_{1} \times D_{2}\right)$ can be almost uniformly approximated by sums of functions of form $x_{1}\left(z_{1}\right) \cdot x_{2}\left(z_{2}\right)$ with $z_{1} \in D_{1}, z_{2} \in D_{2}$ and from the example 1 in [11], p. $89-90$. 
THEOREM 2.1. Let $D$ be a finite connected domain in $C$. Let $Z_{1}$, $Z_{2}, \ldots, Z_{m}$ be components of the complement of $D$. Then

$1^{\circ} \mathscr{H}(D) \approx \mathscr{H}(C)$ if all $Z_{i}$ are points,

$2^{\circ} \mathscr{H}(D) \approx \mathscr{H}\left(C_{0}\right)$ if all $Z_{i}$ are continua,

$3^{\circ} \mathscr{H}(D) \approx \mathscr{H}\left(C_{0}\right) \times \mathscr{H}(C)$ if among $Z_{i}$ there are points and continua.

Proof. For $m=1$ our assertion is obvious. Let us suppose that it is true for an $l$-conected domain. Let $D$ be an m-conected domain. According to Riemann theorem on conform mappings we may suppose that the component $Z_{m}$ of the complement of $D$ is $(\alpha)$ either the point set $\{\infty\}$ or $(\beta)$ the set $\{\tilde{z}:|z| \geqslant 1\}$. Then in both cases there is a positive number $r$ such that

$$
x(z)=\sum_{n=0}^{\infty} a_{n} z^{n}+\sum_{n=1}^{\infty} b_{n} z^{-n} \quad \text { for } \quad x \in \mathscr{H}(D)
$$

and for $|z|>r$ in the case $(\alpha)(1-1 / r<|z|<1$ in the case $(\beta))$. It is easy to see that the correspondence $a \leftrightarrow\left(x_{1}, x_{2}\right)$, where

$$
x_{1}(z)=\sum_{n=0}^{\infty} a_{n} z^{n}, \quad x_{2}(z)=\sum_{n=1}^{\infty} a_{n} z^{n-1},
$$

is an isomorphism between $\mathscr{H}(D)$ and $\mathscr{H}(C) \times \mathscr{H}\left(D \cup Z_{m}\right.$ ) (between $\mathscr{H}(D)$ and $\mathscr{H}\left(C_{0}\right) \times \mathscr{H}\left(D \cup Z_{m}\right)$.

Since the domain $D \cup Z_{m}$ is $m-1$ connected, by the induction hypothesis and lemma 2.2 we obtain the assertion of our theorem, q. e. d.

CONJECTURE. If $D$ is an arbitrary finite connected domain in $C$, then $\mathscr{H}(D)$ is one of the standard forms: $\mathscr{H}(C), \mathscr{H}\left(C_{0}\right), \mathscr{H}\left(C \times C_{0}\right)$.

THEOREM 2.2. Let $D_{1}, \ldots, D_{k}$ be a family of finite connected plane domains. Suppose that all components of each of the spaces $C-D_{i}$ are points for $i=1, \ldots, r$, continua for $i=r+1, \ldots, r+p$, points and continua for $i=r+p+1, \ldots, k$, where $1 \leqslant r \leqslant r+p \leqslant k$. Then $\mathscr{H}\left(D_{1} \times \ldots \times D_{k}\right)$ is isomorphic to the space

$$
\mathscr{H}\left(C^{r} \times C_{0}^{k-r}\right) \times \mathscr{H}\left(C^{r+1} \times C_{0}^{k-r-1}\right) \times \ldots \times \mathscr{H}\left(C^{k-p} \times C_{0}^{p}\right) .
$$

This follows immediately from theorem 2.1 and formulas (17) and (18).

Let $\Omega$ be an algebra with two operations, + and ', and one relation of "equality", defined by the following axioms:

I. $\Omega$ has two generators $u, v$ (in other words, elements of $\Omega$ are sums of mononomials $u^{p} v^{q}$ with $p, q \geqslant 0, p+q>0$, where $u^{p}=u \ldots u, v^{q}=v \ldots v$ times .

II. $\omega_{1}+\omega_{2}=\omega_{2}+\omega_{1}, \quad \omega_{1} \cdot \omega_{2}=\omega_{2} \cdot \omega_{1}, \quad \omega_{1}+\left(\omega_{2}+\omega_{3}\right)=\left(\omega_{1}+\omega_{2}\right)+\omega_{3}$, $\omega_{1} \cdot\left(\omega_{2} \cdot \omega_{3}\right)=\left(\omega_{1} \cdot \omega_{2}\right) \cdot \omega_{3}, \omega_{1} \cdot\left(\omega_{2}+\omega_{3}\right)=\omega_{1} \cdot \omega_{2}+\omega_{1} \cdot \omega_{3}$.

III. If $p^{\prime} \leqslant p, q^{\prime} \leqslant q$, then $u^{p} v^{q}+u^{p^{\prime}} v^{q^{\prime}}=u^{p^{2} v^{q}}$.
Let $\omega=u^{p_{1} v^{q_{1}}}+\ldots+u^{p_{n}} v^{q_{n}}$. Denote by $\omega(X, Y)$ the space construeted from the $\omega$ by substitution $\left(\begin{array}{cccc}\cdot & + & u & v \\ \times & \hat{\hat{\Theta}} & \mathscr{H}(C) & \mathscr{H}\left(C_{0}\right)\end{array}\right)$.

From theorems 2.1, 3.1 and formulas (17) and (18) follows

Theorem 2.3. If $\omega_{1}=\omega_{2}$, then $\omega_{1}(X, Y) \approx \omega_{2}(X, Y)$.

Problem 2.1. Does the relation $\omega_{1}(X, Y)=\omega_{2}(X, X)$ imply $\omega_{1}=\omega_{2}$ ?

Using theorems 2.1, 4.1, results of $\S 5$, and formulas (17), (18) and (19) we obtain the following statements:

$1^{0}$ if degree $\omega_{1} \neq$ degree $\omega_{2}$, then $\omega_{1}(X, Y) \neq \omega_{2}(X, X)$;

20 if $\omega_{1}$ depends on one variable $u, \omega_{2}$ depends on $v$, and $\omega_{3}$ is not equal to any element of $\Omega$ depending only on one variable, then the spaces $\omega_{1}(X, T), \omega_{2}(X, F)$, $\omega_{3}(X, Y)$ are not isomorphic. In particular $\mathscr{H}(C), \mathscr{H}\left(C_{0} \times C\right)$ and $\mathscr{H}\left(C_{0}\right)$ are not isomorphic.

THEOREM 2.4. Let $D=D_{1} \times D_{2} \times \ldots \times D_{k}$ be an arbitrary polycylinder. Then $\mathscr{H}(D)$ is isomorphic to a subspace of $\mathscr{H}\left(C_{0}^{k}\right)$.

Proof. According to (17) and (18) it is sufficient to restrict our attention to the case where $D$ is one-dimensional. By theorem 2.1 we may assume without loss of generality that $\bar{C}-D$ contains at least three points, for instance $\{0\},\{1\},\{\infty\}$. Now by a result of Poincaré ([13], p. 275) there is an analytic function $\varphi$ which transforms $D$ onto $C_{0}$. To complete the proof we apply lemma 2.2 .

THEOREM 2.5. Let $D=D_{1} \times D_{2} \times \ldots \times D_{k}$ be a bounded polycylinder. Then $\mathscr{H}(D)$ contains a subspace isomorphic to $\mathscr{H}\left(C_{0}^{k}\right)$.

Proof. As in the proof of Theorem 2.4, it is enough to restrict our attention to the one-dimension case. $D^{*}$ denotes the complement of the unbounded component of the complement of $D$. Let $r: \mathscr{H}\left(D^{*}\right) \rightarrow \mathscr{H}(D)$ be the restriction of functions in $\mathscr{H}\left(D^{*}\right)$ to functions in $\mathscr{H}(D)$, i. e. $r x(z)=x(z)$ for $z \in D$ and $x \in \mathscr{H}\left(D^{*}\right)$. Obviously $r$ is a linear one-to-one operator and according to the maximum principle $r^{-1}$ is continuous. Hence $\mathscr{H}\left(D^{*}\right)$ is isomorphic to a subspace of $\mathscr{H}(D)$. Since $D^{*}$, is a bounded domain, $\mathscr{H}\left(D^{*}\right)$ is isomorphic to $\mathscr{H}\left(C_{0}\right)$, q. e. d.

From theorems 2.4 and 2.5 we obtain

CoRoLlary 2.1. If $D=D_{1} \times \ldots \times D_{k}$ is a bounded polycylinder, then the spaces $\mathscr{H}(D)$ and $\mathscr{H}\left(C_{0}^{k}\right)$ have the same linear dimension in the sense of Banach ([3], p. 193).

THEOREM 2.6. Let $D_{0}$ be an arbitrary k-dimensional bounded domain. Then, for every $k$-dimensional domain $D, \mathscr{H}(D)$ is isomorphic to a subspace of the space $(\mathscr{H}(D) \times \mathscr{H}(D) \times \ldots)_{s}$.

Proof. There exist points $z^{(n)}$ in $C^{k}$ and positive numbers $s_{n}$ such that

$$
D=\bigcup_{n=1}^{\infty} D_{n}, \quad \text { where } \quad D_{n}=\left\{z \epsilon C^{k}: \frac{z-z^{(n)}}{s^{(n)}} \epsilon D_{0}\right\}
$$


Obviously all the spaces $\mathscr{H}\left(D_{n}\right)$ are isomorphic to $\mathscr{H}(D)$. Let $h_{n}$ denote an isomorphism from $\mathscr{H}\left(D_{n}\right)$ onto $\mathscr{H}\left(D_{0}\right)$ and let $r_{n}: \mathscr{H}(D) \rightarrow$ $\rightarrow \mathscr{H}\left(D_{n}\right)$ be the operation of restriction of functions in $\mathscr{H}(D)$ to functions in $\mathscr{H}\left(D_{n}\right)$. Let us put $h x=\left(h_{n} r_{n} x\right)$ for $x \in \mathscr{H}(D)$. We omit the asy checking that $h$ is the required isomorphism.

COROLLARY 2.2. Let $D$ be an arbitrary k-dimensional domain; then $\mathscr{H}(D)$ is isomorptic to a subspace of the space $\left(\mathscr{H}\left(C_{0}^{k}\right) \times \mathscr{H}\left(C_{0}\right) \times \ldots\right)_{s}$.

\section{§ 3. Matrix representations of spaces $\mathscr{H}_{\mu}(D)$}

1. Matrix form $\mathscr{M}\left(\exp \left(\alpha\left(n_{1}^{q_{1}}+\ldots+n_{r}^{q_{r}}\right)-\frac{1}{\alpha} \quad\left(n_{r+1}^{q r+1}+\ldots+n_{k}^{q_{k}}\right)\right)\right)$. Now we prove

THEOREM 3.1. We have

$$
\mathscr{H}\left(C^{r} \times C_{0}^{k-r}\right) \approx \mathscr{M}\left(\exp \left(\alpha\left(n_{1}+\ldots+n_{r}\right)-\frac{1}{\alpha}\left(n_{r+i}+\ldots+n_{k}\right)\right)\right) .
$$

Proof. The sequence $z^{n}=z_{1}^{n_{1}} \ldots z_{k}^{n_{k}}$ constitutes a basis of the space $\mathscr{H}\left(C^{r} \times C_{0}^{k-r}\right)([10]$, p. 74). We assume as pseudonorms

$$
\|x\|_{\alpha}=\sup \left\{|\infty(z)|:\left|z_{1}\right|, \ldots,\left|z_{r}\right| \leqslant e^{a} ;\left|z_{r+1}\right|, \ldots,\left|z_{k}\right| \leqslant e^{-1 / a}\right\} .
$$

Since $\sum_{n \in \mathscr{S}^{k}}\left\|z^{n}\right\|_{\alpha} /\left\|z^{n}\right\|_{\alpha+1}<\infty$, by Theorem 1.1 we have $\mathscr{H}\left(C^{r} \times C_{0}^{k-r}\right)$ $\approx \mathscr{M}\left(\left\|z^{n}\right\|_{\alpha}\right)$. But $\left\|z^{n}\right\|_{\alpha}=\exp \left(\alpha\left(n_{1}+\ldots+n_{r}\right)-\frac{1}{\alpha}\left(n_{r+1}+\ldots+n_{k}\right)\right)$, q. e. d.

Now we shall establish single matrix representations of the spaces $\mathscr{H}\left(C^{k}\right)$ and $\mathscr{H}\left(C_{0}^{k}\right)$. We shall use the following

LeMma 3.1. Let $\left[\begin{array}{l}j \\ k\end{array}\right]$ denote the number of all mononomials of degree $j$ of $k$ variables. Then $j^{k} / k ! \leqslant\left[\begin{array}{l}j \\ k\end{array}\right] \leqslant(j+1)^{k}$.

Proof. It is easy to see that

$$
\left[\begin{array}{l}
j \\
k
\end{array}\right]=\sum_{p=0}^{j}\left[\begin{array}{c}
p \\
k-1
\end{array}\right] .
$$

Let $f_{k}(t)=\left[\begin{array}{c}\mathrm{E} t \\ k\end{array}\right]$. By (31) we have $\int_{0}^{n+1} f_{k-1}(t) d t=f_{k}(n)$; therefore $\int_{0}^{t} f_{k-1}(\tau) d \tau \leqslant f_{k}(t)$. Hence, by induction, we obtain $f_{k+1}(t) \geqslant t^{k} / k !$; in particular $\left[\begin{array}{c}j \\ k+1\end{array}\right] \geqslant j^{k} / k !$.

On the other hand, from (31) it follows that $\left[\begin{array}{l}j \\ k\end{array}\right] \leqslant(j+1)\left[\begin{array}{c}j \\ k-1\end{array}\right]$, and by induction $\left[\begin{array}{l}j \\ k\end{array}\right] \leqslant(j+1)^{k-1}$, q. e.d.

TheOREM $3.1^{\prime} . \mathscr{H}\left(C^{k}\right) \approx \mathscr{M}(\exp a \sqrt[k]{n})$ and $\mathscr{H}\left(C_{0}^{k}\right) \approx \mathscr{M}(\exp (-\sqrt[k]{n} / a))$.
Proof. Let $\boldsymbol{p}(n)=\left(p_{1}(n), \ldots, p_{k}(n)\right)$ be an arbitrary one-to-one function from $\mathscr{N}^{1}$ onto $\mathscr{N}^{k}$ such that $|\boldsymbol{p}(n)| \leqslant\left|\boldsymbol{p}\left(n^{\prime}\right)\right|$ for $n \leqslant n^{\prime}$, where $|\boldsymbol{p}(n)|=p_{1}(n)+\ldots+p_{k}(n)$. Write $e_{n}=z_{1}^{p_{1}(n)} \ldots z_{k}^{p_{k}(n)}$. By Lemma 3.1, there are positive constants $A$ and $B$ such that

$$
\begin{gathered}
\exp \alpha A \sqrt[k]{n} \leqslant\left\|e_{n}\right\|_{\alpha} \leqslant \exp \alpha B \sqrt[k]{n} \quad \text { for } \quad \mathscr{H}\left(C^{k}\right), \\
\exp (-A \sqrt[k]{n} / \alpha) \leqslant\left\|e_{n}\right\|_{a} \leqslant \exp (-B \sqrt[k]{n} / \alpha) \text { for } \mathscr{H}\left(C_{0}^{k}\right) .
\end{gathered}
$$

Thus the formula $T\left(c_{n}\right)=\sum_{n \in \mathcal{N}^{1}} c_{n} z^{p(n)}$ gives the required isomorphic mappings from $\mathscr{M}(\exp a \sqrt[k]{n})$ onto $\mathscr{H}\left(C^{k}\right)$ and from $\mathscr{M}(\exp (-\sqrt[k]{n} / \alpha))$ onto $\mathscr{H}\left(C_{0}^{k}\right)$, q. e. d.

The next two theorems will concern matrix representations of spaces of holomorphic functions defined on circular or $p_{1}, \ldots, p_{k}$-circular $([10]$, p. 113 and 117 ) domains $D$. For simplicity we shall formulate these theorems for the case where the centre of $D$ coincides with $(0, \ldots, 0)$.

THEOREM 3.2. Let $D$ be a bounded domain in $C^{k}$ such that

$$
\overline{a D} \subset D \quad \text { for every } \quad|a|<1\left(^{8}\right) \text {. }
$$

Then $\mathscr{H}(D) \approx \mathscr{M}(\exp (-\sqrt[k]{n} / \alpha))$

Proof. Let $D_{\varepsilon}=(1-\varepsilon) D$. We introduce scalar-products

$$
(x, y)_{\varepsilon}=\int_{D_{\varepsilon}} x\left(z_{1}, \ldots, z_{k}\right) \overline{y\left(z_{1}, \ldots, z_{k}\right)} d x_{1} \ldots d x_{k} d y_{1} \ldots d y_{k},
$$

where $z_{j}=x_{j}+i y_{j}$ for $j=1, \ldots, k$, and the integral is taken over the domain $D$ in a $2 k$-dimensional real Euclidean space ([10], p. 119).

The pseudonorms $\| x \| _ { \varepsilon } = 1 \longdiv { ( x , x ) _ { \varepsilon } } \quad ( 0 < \varepsilon < 1 )$ give a topology equivalent to the almost uniform convergence, because

$$
\left|D_{\varepsilon}\right|^{-1}|| x \|_{\varepsilon} \leqslant \sup _{z_{\varepsilon} D_{\varepsilon}}|x(z)| \leqslant \pi^{-1} r_{\varepsilon \varepsilon^{\prime}}^{-k}|x|_{\varepsilon^{\prime}},
$$

where $\left|D_{\varepsilon}\right|$ is the volume of $D_{\varepsilon}$ and $r_{\varepsilon \varepsilon^{\prime}}$, is the distance between the set $D_{\varepsilon}$ and the complement of $D_{\varepsilon^{\prime}}([10]$, p. 120).

For the scalar product $(x, y)_{0}$ we can construct an orthonormal basis $\left(e_{n}\right)$ constituted from homogeneous polynomials ([10], p. 132). The number of elements of the basis which are polynomials of degree $j$ is equal to $\left[\begin{array}{l}j \\ k\end{array}\right]$. Since $e_{n}$ are homogeneous polynomials, we have $\left(e_{r}, e_{s}\right)_{\varepsilon}=0$ for $r \neq s,\left(e_{r}, e_{r}\right)_{\varepsilon}=(1-\varepsilon)^{j}\left\|e_{r}\right\|_{0}$, where $j$ is the degree of the polynomial $e_{r}$. Let $\varepsilon_{\alpha}=1-\exp (-1 / a)$. Then $\left\|e_{n}\right\|_{\varepsilon_{\alpha}}=\left\|e_{n}\right\|_{0} \exp (-j / \alpha)$,

$\left.{ }^{8}\right) \bar{A}$ denotes the closure of the set $A$. 
where $j$ is the degree of the polynomial $e_{n}$. Now, applying Theorem 1.1 and Lemma 3.1, we obtain $\mathscr{H}(D) \approx \mathscr{M}(\exp (-\sqrt[k]{n+k} / \alpha))$. But this space is trivially isomorphic to the space $\mathscr{M}(\exp (-\sqrt[k]{n} / \alpha))$, q. e. d.

THEOREM 3.3. Let $D$ be a bounded domain in $C^{k}$ such that (33) there are positive integers $p_{1}, \ldots, p_{k}$ such that for every real $t$

$$
\left\{\left(z_{1} \exp i p_{1} t, \ldots, z_{k} \exp i p_{k} t\right):\left(z_{1}, \ldots, z_{k}\right) \in D\right\} \subset D,
$$

$$
\overline{t D} \subset D \text { for each } 0<t<1 \text {. }
$$

Then $\mathscr{H}(D) \approx \mathscr{M}(\exp (-\sqrt[k]{n} / \alpha))$.

Proof. Let $\Phi(z)=\left(z_{1}^{p_{1}}, \ldots, z_{k}^{p_{k}}\right)$. It follows from (33) and (34) that the domain $D^{*}=\Phi^{-1}(D)$ fulfills condition (32). Let us assign to any function $x(z) \in \mathscr{H}(D)$ the function $(U x)(z)=x(\Phi(z))$ with $z \in D^{*}$. It is easy to verify that $U$ is an isomorphic mapping from $\mathscr{H}(D)$ onto a subspace $X$ of the space $\mathscr{H}\left(D^{*}\right)$.

In the same way as in the proof of Theorem 3.2 we can construct a basis of $X$ chosen from the homogeneous polynomials. The elements of this basis are sums of mononomials $z_{1}^{n_{1}} \ldots z_{k}^{n_{k}}$ such that $n_{i}$ is divisible by $p_{i}$ for $i=1,2, \ldots, k$. Let $\tau_{j}$ denote the number of such mononomials of degree not greater than $j$. As in Lemma 3.1 we can prove that there are positive constants $A$ and $B$ such that $A j^{k} \leqslant s_{j} \leqslant B j^{k}$. From this inequality follows the assertion of the theorem.

THEOREM 3.4. Let $\mu(\varepsilon, z)=\exp \left(-\sum_{j=1}^{k}\left(\tau_{j}+\varepsilon\right)\left|z_{j}\right|^{p_{j}}\right), \quad \tau_{1}, \ldots, \tau_{r}=0$, $\tau_{r+1}, \ldots, \tau_{k}>0, p_{j}>0 ;$ then $\mathscr{H}_{\mu} \approx \mathscr{M}\left(\exp \left(\alpha\left(n_{1}+\ldots+n_{r}\right)-\frac{1}{\alpha}\left(n_{r+1}+\right.\right.\right.$ $\left.\left.+\ldots+n_{k}\right)\right)$.

The isomorphic mapping from $\mathscr{H}_{\mu}$ onto $\mathscr{M}\left(\exp \left(\alpha\left(n_{1}+\ldots+n_{r}\right)-\right.\right.$ $\left.\left.\frac{1}{a}\left(n_{r+1}+\ldots+n_{k}\right)\right)\right)$ is of the form

(35) $T \sum_{\boldsymbol{n} \in \mathcal{W}^{k}} c_{\boldsymbol{n}} z^{\boldsymbol{n}}=\left(d_{\boldsymbol{n}} \boldsymbol{c}_{\boldsymbol{n}}\right)$, where $d_{\boldsymbol{n}}=\left(\prod_{j=1}^{k}\left(n_{j} / p_{j}\right)^{n_{j} / n_{j}}\right) \exp \left(-\sum_{j=1}^{k} n_{j} / p_{j}\right)$.

Proof. We have

$$
\begin{aligned}
& \left\|z^{\boldsymbol{n}}\right\|_{\varepsilon}=\max _{z_{\varepsilon} C^{n}}\left|z^{\boldsymbol{n}}\right| \exp \left(-\sum_{j=1}^{k}\left(\tau_{j}+\varepsilon\right)\left|z_{j}\right|^{p_{j}}\right) \\
& \quad=\max _{t_{j}>0} \exp \left(\sum_{j=1}^{k} n_{j} \log t_{j}-\sum_{j=1}^{k}\left(\tau_{j}+\varepsilon\right) t_{j}^{p_{j}}\right) .
\end{aligned}
$$

$$
f\left(t_{1}, \ldots, t_{k}\right)=\sum_{j=1}^{k} n_{j} t_{j}-\sum_{j=1}^{k}\left(\tau_{j}+\varepsilon\right) t_{j}^{p_{j}}
$$

Solving the equations $\partial f / \partial t_{j}=0$ we find that the function $f$ has its maximum for $t_{j}=\sqrt[n_{j}]{n_{j} /\left(p_{j}\left(\tau_{j}+\varepsilon\right)\right)}, j=1, \ldots, k$, whence

$$
\left\|z^{n}\right\|_{\varepsilon}=\left(\prod_{j=1}^{k}\left(\frac{n_{j} / p_{j}}{\tau_{j}+\varepsilon}\right)^{p_{j} / p_{j}}\right) \exp \left(-\sum_{j=1}^{k} n_{j} / p_{j}\right) .
$$

Thus the formula $U \sum_{n \in \mathscr{K}^{k}} c_{n} z^{n}=\left(c_{n}\right)$ gives an isomorphic mapping from $\mathscr{H}_{\mu}$ onto $\mathscr{M}\left(d_{n} \exp \left(\alpha\left(n_{1}+\ldots+n_{r}\right)-\frac{1}{\alpha}\left(n_{r+1}+\ldots+n_{k}\right)\right)\right)$, where $d_{n}$ are given by formula (35). This is equivalent to the assertion of the theorem.

THEOREM 3.5. Let $\mu(\varepsilon, z)=\exp \left(-\left.\left.\left.\sum_{j=1}^{k}\left(\tau_{j}+\varepsilon\right)|\log | z\right|_{j}\right|_{j}\right|_{j}\right)$, where $p_{j}>1$, $\tau_{1}, \ldots, \tau_{r}=0, \tau_{r+1}, \ldots, \tau_{k}>0$, and let $q_{j}=p_{j} /\left(p_{j}-1\right), j=1, \ldots, k$. Then $\mathscr{H}_{\mu} \approx \mathscr{M}\left(\exp \left(\alpha\left(n_{1}^{q_{1}}+\ldots+n_{r}^{q_{r}}\right)-\frac{1}{\alpha}\left(n_{r+1}^{q_{r+1}}+\ldots+n_{k}^{q_{k}}\right)\right)\right)$.

Proof. In the same way as in the preceding proof we obtain

$$
\left|z^{n}\right|_{\varepsilon}=\prod_{j=1}^{k} A_{\varepsilon j}^{n_{j j}^{q_{j}}}, \quad \text { where } \quad A_{\varepsilon j}=\exp \left(\left(\frac{1}{\left(\tau_{j}+\varepsilon\right) p_{j}}\right)^{q_{j}} q_{j}^{-1}\right) .
$$

If $\tau_{j}=0$, then $\lim _{\varepsilon \rightarrow 0} A_{\varepsilon j}=\infty$; if $\tau_{j}>0$, then $\lim _{\varepsilon \rightarrow 0} A_{\varepsilon j}=A_{j}$. From Theorem 1.6 it follows that $\mathscr{H}_{\mu} \approx \mathscr{\sim}\left(\left|z^{n}\right|_{\varepsilon_{a}}\right)$, where $\underset{\substack{\varepsilon \rightarrow 0 \\\left(\varepsilon_{\alpha}\right)}}{ }$ is an arbitrary sequence tending to zero. The transformation

$$
U\left(\xi_{\boldsymbol{n}}\right)=\left(d_{\boldsymbol{n}} \xi_{\boldsymbol{n}}\right), \quad \text { where } \quad d_{\boldsymbol{n}}=\prod_{j=r+1}^{k} A_{j}^{n_{j}^{q_{j}}} \quad \text { for } \quad \boldsymbol{n} \in \mathcal{N}^{k},
$$

maps this space onto the space given in the assertion of Theorem 3.5.

Remark 3.1. The space $\mathscr{H}_{\mu}$ considered in Theorem 3.5 is isomorphic to the space $F_{p \tau}^{k}$ of all holomorphic functions $x(z)$ which are periodic with the period $2 \pi$ with respect to every variable and such that

$$
\|x\|_{\varepsilon}=\sup |x(z)| \exp \left(-\sum_{j=1}^{k}\left(\tau_{j}+\varepsilon\right)\left|\operatorname{Im} z_{j}\right|^{p_{j}}\right)<\infty .
$$

The isomorphic mapping from $\mathscr{H}_{\mu}$ onto $F_{p \tau}^{k}$ is given by the formula $(U x)(z)=x\left(\exp i z_{1}, \ldots, \exp i z_{k}\right)$.

THEOREM 3.6. Let

$$
\mu(\varepsilon, z)=\exp \left(-\varepsilon \sum_{j=1}^{k}\left(\log \frac{1}{\left|z_{j}\right|}\right)^{-s_{j}}\right), \quad s_{j}>0 .
$$


Then

$$
\mathscr{H}_{\mu}\left(C_{0}^{k}\right) \approx \mathscr{M}\left(\exp \left(-\frac{1}{\alpha} \sum_{j=1}^{k} n_{j}^{q_{j}}\right)\right), \quad \text { where } \quad q_{j}=s_{j} /\left(1+s_{j}\right)
$$

This isomorphism is realized by the transformation $T\left(\sum_{\mathbf{n} \in \mathcal{H}^{k}} c_{\mathbf{n}^{n}} z^{n}\right)=\left(c_{\boldsymbol{n}}\right)$.

Proof. We have

$$
\left\|z^{n}\right\|_{\varepsilon}=\exp \left(-\sum_{j=1}^{k}\left(\frac{n_{j}}{\varepsilon s_{j}}\right)^{-1 /\left(s_{j}+1\right)}\left(n_{j}+\varepsilon\right)\right) .
$$

Hence if $|\boldsymbol{n}|$ is sufficiently large, then

$$
\exp \left(-2 \max _{x}\left(\varepsilon s_{j}\right)^{-1 / s_{j}+1} \sum_{j=1}^{k} n_{j}^{q_{j}} \leqslant\left\|z^{n}\right\|_{\varepsilon} \leqslant \exp \left(-\frac{1}{2} \min _{j}\left(\varepsilon_{j} s\right)^{-1 / s_{j}+1} \sum_{j=1}^{k} n_{j}^{q_{j}}\right) .\right.
$$

This inequality gives us the assertion of the theorem $\left({ }^{9}\right)$.

Remark 3.2. Let $\mu(\varepsilon, z)$ be the function defined in Theorem 3.6 and let $\mu_{1}(\varepsilon, z)=\exp \left(-\varepsilon \sum_{j=1}^{k}\left(1-\left|z_{j}\right|\right)^{-s_{j}}\right)$. Then $\mathscr{H}_{\mu}=\mathscr{H}_{\mu_{1}}$.

This follows immediately from the fact that

$$
\lim _{t \rightarrow 1-.} \frac{1 /(1-t)}{1 / \log (1 / t)}=1 \text {. }
$$

2. Matrix form $\mathscr{M}\left(\left(n_{1}^{n_{1}} \ldots n_{r}^{n_{r}}\right)^{\alpha} \cdot\left(n_{r+1}^{n_{r}+1} \ldots n_{k}^{n_{k}}\right)^{-1 / a}\right)$. Now we prove THEOREM 3.7. Let $\mu(\varepsilon, z)=\exp \left(-\sum_{j=1}^{k}\left|z_{j}\right|^{p_{j}+\varepsilon}\right)$, where $p_{1}, \ldots, p_{r}=0$, $p_{r+1}, \ldots, p_{k}>0$. Then $\mathscr{H}_{\mu} \approx \mathscr{M}\left(\left(n_{1}^{n_{1}} \ldots n_{r}^{n}\right)^{\alpha} \cdot\left(n_{r+1}^{n_{r+1}} \ldots n_{k}^{n_{k}}\right)^{-1 / \alpha}\right)$.

Proof. We have

$$
\left\|z^{\mathbf{n}}\right\|_{\varepsilon}=\prod_{j=1}^{k} n_{j}^{\mathbf{n}_{j} /\left(p_{j}+\bar{\varepsilon}\right)}\left(p_{j}+\varepsilon\right)^{-n_{j} /\left(p_{j}+\varepsilon\right)} \cdot \exp \left(-n_{j} /\left(p_{j}+\varepsilon\right)\right) .
$$

Hence for arbitrary $\eta>0$

$$
\prod_{j=1}^{k} n_{j}^{n_{j} /\left(p_{j}+\varepsilon+\eta\right)} \leqslant\left\|z^{n}\right\|_{\varepsilon} \leqslant \prod_{j=1}^{k} n_{j}^{n_{j} /\left(p_{j}+\varepsilon-\eta\right)}
$$

for sufficiently large $|\boldsymbol{n}|$. Therefore $\mathscr{H}_{\mu} \approx \mathscr{M}\left(\prod_{j=1}^{k} n_{j}^{n_{j} /\left(p_{j}+1 / a\right)}\right)$, by Theo-

$\left({ }^{9}\right)$ It is enough to notice that Theorem 1.6 holds true if we replace spaces $\mathscr{H}_{\mu}(O)$ by $\mathscr{H}_{\mu}\left(C_{0}\right)$. rem 1.6. But this space may be isomorphically mapped onto the space given in the assertion of the theorem by the transformation

$$
T\left(c_{\boldsymbol{n}}\right)=\left(d_{\boldsymbol{n}} c_{\boldsymbol{n}}\right), \quad \text { where } \quad \boldsymbol{d}_{\boldsymbol{n}}=\prod_{j=r+1}^{k} n_{j}^{n_{j} / p_{j}} .
$$

3. Matrix form $\mathscr{M}\left(\exp \left(n_{1}^{\alpha}+\ldots+n_{r}^{\alpha}+n_{r+1}^{q_{r+1}-1 / \alpha}+\ldots+n_{k}^{q_{k}-1 / \alpha}\right)\right)$. Now we prove.

THEOREM 3.8. Let $\mu(\varepsilon, z)=\exp \left(-\sum_{j=1}^{k} \log \left|z_{j}\right|^{p_{j}+\varepsilon}\right)$, where $p_{1}, \ldots, p_{r}=1$, $p_{r+1}, \ldots, p_{k}>1$. Then $\mathscr{H}_{\mu} \approx \mathscr{M}\left(\exp \left(n_{1}^{a}+\ldots+n_{r}^{\alpha}+n_{r+1}^{q_{r+1}-1 ; a}+\ldots+n_{k}^{q_{k}-1 / a}\right)\right)$, $q_{j}=p_{j} /\left(p_{j}-1\right)$

Proof. We have

$$
\left\|z^{\boldsymbol{n}}\right\|_{\varepsilon}=\prod_{j=1}^{k} \exp \left(\left(n_{i j} /\left(p_{j}+\varepsilon\right)\right)^{\left(p_{j}+\varepsilon\right) /\left(p_{j}+\varepsilon-1\right)} \cdot\left(p_{j}+\varepsilon-1\right) /\left(p_{j}+\varepsilon\right)\right) .
$$

Hence, for every $\eta>0$,

$$
\prod_{j=1}^{k} \exp n_{j}{ }^{\left(p_{j+\varepsilon}+\eta\right) /\left(p_{j+\varepsilon-\eta-1)}\right.} \leqslant\left\|z^{\mathbf{n}}\right\|_{\varepsilon} \leqslant \exp n_{j}{ }^{\left(p_{j}+\varepsilon+\eta\right) /\left(p_{j}+\varepsilon+\eta-1\right)}
$$

for sufficiently large $|\boldsymbol{n}|$. Hence, by Theorem 1.6, we obtain the assertion of our theorem.

\section{§ 4, Approximative dimension}

1. Space $\mathscr{M}\left(\exp \left(\alpha\left(n_{1}^{q_{1}}+\ldots+n_{r}^{q_{r}}\right)-\frac{1}{\alpha}\left(n_{r+1}^{q_{r+1}}+\ldots+n_{k}^{q_{k}}\right)\right)\right)$. Now we prove

THEOREMI 4.1. Let $X \approx \mathscr{M}\left(\exp \left(\alpha\left(n_{1}^{\alpha_{3}}+\ldots+n_{r}^{q_{r}}\right)-\frac{1}{\alpha}\left(n_{r+1}^{q_{r+1}}+\ldots+\right.\right.\right.$ $\left.\left.\left.+n_{k}^{q_{k}}\right)\right)\right)$, with $q_{i}>0 ; s=\sum_{i=1}^{k} q_{i}^{-1}$. Then

$$
\Phi(X)=\left\{\begin{array}{l}
\left\{\varphi: \lim \left(\log \frac{1}{\varepsilon}\right)^{s+1} / \log \varphi(\varepsilon)=0\right\}, \quad \text { if } \quad r=0, \\
\left\{\varphi: \varlimsup \lim \left(\log \frac{1}{\varepsilon}\right)^{s+1} / \log \varphi(\varepsilon)<\infty\right\}, \quad \text { if } \quad r>0 .
\end{array}\right.
$$

Proof. Put $a_{a n}^{(j)}=\exp \left(a n^{q_{j}}\right)$ for $j=1, \ldots, r, a_{a n}^{(j)}=\exp \left(-n^{q_{j}} / \alpha\right)$ for $j=r+1, \ldots, k$. Since, for any $q>0$,

$$
\exp \left(\alpha n^{q}\right) / \exp \left(\beta n^{q}\right) \geqslant \varepsilon \text { if and only if } n \leqslant\left(\frac{1}{\beta-\alpha} \log \frac{1}{\varepsilon}\right)^{1 / q}
$$


$\exp \left(-\frac{n^{q}}{\alpha}\right) / \exp \left(-\frac{n^{q}}{\beta}\right) \geqslant \varepsilon$ if and only if $n \leqslant\left((1 / \alpha-1 / \beta)^{-1} \log \frac{1}{\varepsilon}\right)^{1 / q}$,

we obtain

$$
N_{a \beta j}(\varepsilon)= \begin{cases}1+\mathrm{E}\left(\frac{1}{\beta-\alpha} \log \frac{1}{\varepsilon}\right)^{1 / q} \quad \text { for } \quad j=1, \ldots, r, \\ 1+\mathrm{E}\left((1 / a-1 / \beta)^{-1} \log \frac{1}{\varepsilon}\right)^{1 / q} \quad \text { for } \quad j=r+1, \ldots, k .\end{cases}
$$

Hence, for sufficiently small $\varepsilon$,

and

$$
\prod_{j=1}^{k} N_{\alpha \beta j}(\sqrt[2 k]{\varepsilon}) \geqslant \frac{1}{2 k}(\beta-\alpha)^{-s^{\prime}}(1 / \alpha-1 / \beta)^{-s^{\prime \prime}}\left(\log \frac{1}{\varepsilon}\right)^{s}
$$

$$
\prod_{j=1}^{k} N_{\alpha \beta j}(\varepsilon) \leqslant 2^{k}(\beta-\alpha)^{-s^{\prime}}(1 / \alpha-1 / \beta)^{s^{\prime \prime}}\left(\log \frac{1}{\varepsilon}\right)^{s}
$$

where $s^{\prime}=1 / q_{1}+\ldots+1 / q_{r}, s^{\prime \prime}=1 / q_{r+1}+\ldots+1 / q_{k}, s=s^{\prime}+s^{\prime \prime}$. Thus, according to Theorem 1.3, for sufficiently small $\varepsilon$ we have

$$
\text { (41) } \begin{aligned}
\frac{1}{2 k}(\beta-\alpha)^{-s^{\prime}}(1 / \alpha-1 / \beta)^{s^{\prime \prime}} & \left(\log \frac{1}{\varepsilon}\right)^{s+1} \\
& \leqslant \log M_{\alpha \beta}(\varepsilon) \\
& \leqslant 2^{k+1}(\beta-\alpha)^{-s^{\prime}}(1 / \alpha-1 / \beta)^{s^{\prime \prime}}\left(\log \frac{1}{\varepsilon}\right)^{s+1} .
\end{aligned}
$$

In virtue of the definition of $M(A, B), \varphi \in M\left(K_{\beta}, K_{a}\right)\left({ }^{1}\right)$ if and only if there exists $\varepsilon_{0}>0$ such that

$$
\log \varphi(\varepsilon) \geqslant \log M_{a \beta}(X ; \varepsilon) \quad \text { for every } \quad \varepsilon<\varepsilon_{0} .
$$

Hence, by (41),

implies

$$
2^{k+1} \varlimsup_{\varepsilon \rightarrow 0}\left((\beta-\alpha)^{-s^{\prime}}(1 / \alpha-1 / \beta)^{s^{\prime \prime}}\left(\log \frac{1}{\varepsilon}\right)^{s}\right) / \log \varphi(\varepsilon)<1
$$

which implies

$$
\varphi \in M\left(K_{\beta}, K_{\alpha}\right)
$$

$$
\frac{1}{2 k} \varlimsup_{\varepsilon \rightarrow 0}\left((\beta-\alpha)^{-\delta^{\prime}}(1 / \alpha-1 / \beta)^{s^{\prime \prime}}\left(\log \frac{1}{\varepsilon}\right)^{s}\right) / \log \varphi(\varepsilon)<1 .
$$

Now Theorem 1.2 gives us the assertion of the theorem.

COROLLART 4.1. If $0<r \leqslant k$, then $\mathrm{d}_{a} \mathscr{H}\left(C^{r} \times C_{0}^{k-r}\right)=\mathrm{d}_{a} \mathscr{H}\left(C^{k}\right)<$ $<\mathrm{d}_{\alpha} \mathscr{H}\left(C_{0}^{k}\right)$.

(') $K_{\alpha}=\left\{x:\|x\|_{\alpha}<1\right\}, K_{\beta}=\left\{x:\|x\|_{\beta}<1\right\}$.
CoROLLARY 4.2. If $\mathrm{d}_{a} \mathscr{H}(D)=\mathrm{d}_{a} \mathscr{H}\left(D_{1}\right)$, then $\operatorname{dim} D=\operatorname{dim} D_{1}$.

COROLLARY 4.3. If $D$ is an arbitrary bounded domain of the k-dimensional Euclidean. complex space, then.

$$
\Phi(\mathscr{H}(D))=\left\{\varphi: \lim _{\varepsilon \rightarrow 0}\left(\log \frac{1}{\varepsilon}\right)^{k+1} / \log \varphi(\varepsilon)=0\right\} .
$$

These corollaries follow from Theorems 1.5, 2.4, 2.5 and 2.6.

2. Space $\mathscr{M}\left(\left(n_{1}^{n_{1}} \ldots n_{r}^{n_{r}}\right)^{\alpha} \cdot\left(n_{r+1}^{n_{r+1}} \ldots n_{k}^{n_{k}}\right)^{-1 / \alpha}\right)$. Now we prove

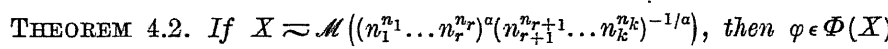
if and only if

$$
\varlimsup_{\varepsilon \rightarrow 0} \frac{\left(\log \frac{1}{\varepsilon}\right)^{k+1} /\left(\log \log \frac{1}{\varepsilon}\right)^{k}}{\log \varphi(\varepsilon)}\left\{\begin{array}{l}
=0 \quad \text { in the case where } r=0 \\
<+\infty \text { in the case where } r>0
\end{array}\right.
$$

Proof. Put $a_{n}^{(j)}=n^{\alpha n}$ for $j=1, \ldots, r, a_{n}^{(j)}=n^{-n / \alpha}$ for $j=r, \ldots, k$. It is easy to verify that

$$
N_{\alpha \beta j}(\varepsilon)= \begin{cases}\mathrm{E} \varkappa\left(\frac{1}{\beta-\alpha} \log \frac{1}{\varepsilon}\right)+1 \quad \text { for } \quad j \leqslant r, \\ \mathrm{E} \varkappa\left((1 / \alpha-1 / \beta)^{-1} \log \frac{1}{\varepsilon}\right)+1 \text { for } j>r,\end{cases}
$$

where $x(t)$ is the function inverse to the function $f(t)=t \log t$.

Since

$$
\lim _{t \rightarrow 0} \frac{x(t)}{t / \log t}=1
$$

for sufficiently small $\varepsilon$, we have $\frac{1}{2} A_{a \beta j} \log \frac{1}{\varepsilon} / \log \log \frac{1}{\varepsilon} \leqslant N_{a \beta j}(\varepsilon) \leqslant$ $2 A_{\alpha \beta j} \log \frac{1}{\varepsilon} / \log \log \frac{1}{\varepsilon}$, where $A_{\alpha \beta j}=1 /(\beta-\alpha)$ for $j=1, \ldots, r, A_{\alpha \beta j}=$ $(1 / \alpha-1 / \beta)^{-1}$ for $j=r+1, \ldots, k$. Therefore, for sufficiently small $\varepsilon$,

and

$$
\prod_{j=1}^{k} N_{\alpha \beta j}(\sqrt[2 k]{\varepsilon}) \geqslant\left(\frac{1}{2}\right)^{3 k^{2}}(\beta-\alpha)^{-r}(1 / \alpha-1 / \beta)^{r-k}\left(\log \frac{1}{\varepsilon} / \log \log \frac{1}{\varepsilon}\right)^{k}
$$

$$
\prod_{j=1}^{k} N_{a \beta j}(\varepsilon) \leqslant 2^{k}(\beta-\alpha)^{-r}(1 / \alpha-1 / \beta)^{r-k}\left(\log \frac{1}{\varepsilon} / \log \log \frac{1}{\varepsilon}\right)^{k} .
$$

Now in the same way as in the preceding section one can deduce the assertion of the theorem. 
3. Space $\mathscr{M}\left(\exp \left(n_{1}^{\alpha}+\ldots+n_{r}^{\alpha}+n_{r+1}^{q_{r+1}-1 / \alpha}+\ldots+n_{k}^{q_{k}-1 / \alpha}\right)\right)$. We now prove then

THEOREM 4.3. If $X \approx \mathscr{M}\left(\exp \left(n_{1}^{\alpha}+\ldots+n_{r}^{\alpha}+n_{r+1}^{q_{r+1}-1 / \alpha}+\ldots+n_{k}^{q_{k}-1 / \alpha}\right)\right.$,

$$
\Phi(X)=\left\{\varphi: \lim _{\varepsilon \rightarrow 0}(\log 1 / \varepsilon)^{s+1+\eta} / \log \varphi(\varepsilon)=0 \text { for some } \eta>0\right\},
$$

.where $s=1 / q_{r+1}+\ldots+1 / q_{k}$.

Proof. Let $a_{n}^{(j)}=\exp n^{\alpha}$ for $j=1, \ldots, r, a_{n}^{(j)}=\exp n^{a_{j}-1 / \alpha}$ for $j=r+1, \ldots, k$. It is easily seen that, for $\alpha<\beta$,

$$
\frac{\exp n^{\alpha}}{\exp n^{\beta}} \geqslant \varepsilon \text { if and only if } n \leqslant \lambda_{\alpha \beta}\left(\log \frac{1}{\varepsilon}\right),
$$

and

$$
\frac{\exp n^{q-1 / \alpha}}{\exp n^{q-1 / \beta}} \geqslant \varepsilon \text { if and only if } n \leqslant \lambda_{q-\frac{1}{\sigma^{\prime}}, q_{-\frac{1}{\beta}}}\left(\log \frac{1}{\varepsilon}\right)
$$

where $\lambda_{\alpha \beta}(t)$ is the function inverse to the function $f(t)=t^{\beta}-t^{\alpha}(\alpha<\beta)$. Hence

$$
N_{a \beta j}(\varepsilon)=\left\{\begin{array}{lll}
\mathrm{E} \lambda_{a \beta}\left(\log \frac{1}{\varepsilon}\right) & \text { for } & j \leqslant r, \\
\mathrm{E} \lambda_{a_{j}-\frac{1}{a}, a_{j}-\frac{1}{\beta}}\left(\log \frac{1}{\varepsilon}\right) & \text { for } & j>r .
\end{array}\right.
$$

Since $\lim \lambda_{a \beta}(t) / t^{1 / \beta}=1$, we obtain, for sufficiently small $\varepsilon$,

$$
N_{a \beta j}(\sqrt[2 k]{\varepsilon}) \geqslant \frac{1}{4 k}\left(\log \frac{1}{\varepsilon}\right)^{1 / \beta}, \quad N_{\alpha \beta j}(\varepsilon) \leqslant 2\left(\log \frac{1}{\varepsilon}\right)^{1 / \beta} \text { for } \quad j \leqslant r
$$

and

$$
\begin{aligned}
& N_{\alpha \beta j}(\sqrt[2 k]{\varepsilon}) \geqslant(1 / 2)(2 k)^{-1 / \alpha_{j}}\left(\log \frac{1}{\varepsilon}\right)^{\left(a_{j}-1 / \beta\right)^{-1}}, \\
& N_{\alpha \beta_{j}}(\varepsilon) \leqslant 2\left(\log \frac{1}{\varepsilon}\right)^{\left(a_{j}-1 / \beta\right)^{-1}} \quad \text { for } j>r .
\end{aligned}
$$

Therefore, according to Theorem 3.1, we obtain

$A_{k}\left(\log \frac{1}{\varepsilon}\right)^{\left(r / \beta+\Sigma\left(q_{j}-1 / \beta\right)^{-1}\right)+1} \leqslant M_{\alpha \beta}(\varepsilon) \leqslant B_{k}\left(\log \frac{1}{\varepsilon}\right)^{\left(r / \beta+\Sigma\left(q_{j}-1 / \beta\right)^{-1}\right)_{+1}}$,

where $A_{k}$ and $B_{k}$ are constants.

From the last formula follows the assertion of Theorem 4.3, because

$$
\lim _{\beta \rightarrow \infty}\left(r / \beta+\sum\left(q_{j}-1 / \beta\right)^{-1}\right)=s .
$$

\$ 5. Power spaces. Single matrix representations. Examples of non-isomorphic spaces having the same approximative dimension

1. Definition. Nuclear Köthe spaces of the forms $\mathscr{M}\left(a_{n}^{a}\right)$ and $\mathscr{M}\left(b_{n}^{1 / a}\right)$ will be called power spaces of infinite or finite type, respectively.

From Theorem 1.1 it follows that in the case of power spaces

(51) There exist $\beta>0$ such that $\sum_{n} a_{n}^{\beta}<\infty$; for every $t>0, \sum_{n} b_{n}^{t}<\infty$.

Without loss of generality it may be assumed that

$$
1 \leqslant a_{0} \leqslant a_{1} \leqslant \ldots ; \quad 1 \geqslant b_{0} \geqslant b_{1} \geqslant \ldots
$$

Let $\varphi(\varepsilon)$ and $\psi(\varepsilon)$ be functions defined for $\varepsilon>0$. If there exists $\varepsilon_{0}>0$ such that $\varphi(\varepsilon) \geqslant \psi(\varepsilon)$ (resp. $\varphi(\varepsilon)>\psi(\varepsilon)$ ), for $\varepsilon<\varepsilon_{0}$ we shall write

$$
\varphi(\varepsilon) \geqslant \psi(\varepsilon) \quad(\text { resp. } \varphi(\varepsilon) \dot{>} \psi(\varepsilon)) .
$$

Let $X=\mathscr{M}\left(a_{n}^{a}\right), Y=\mathscr{M}\left(b_{n}^{1 / \alpha}\right)$, with $\left(a_{n}\right)$ and $\left(b_{n}\right)$ satisfying conditions (51) and (52). Put

$$
M_{a}(X ; \varepsilon)=\prod_{n=0}^{\infty} \mathrm{E}\left(1+1 /\left(\varepsilon a_{n}^{a}\right)\right), \quad \tilde{M}_{a}(Y ; \varepsilon)=\prod_{n=0}^{\infty} \mathrm{E}\left(1+b_{n}^{1 / \alpha} / \varepsilon\right),
$$

and

$$
M_{a}(X)=\left\{\varphi: \varphi(\varepsilon) \geqslant M_{a}(X ; \varepsilon)\right\}, \quad \tilde{M}_{a}(Y)=\{\varphi: \varphi(\varepsilon) \geqslant \tilde{M}(Y ; \varepsilon)\}
$$

for $a=1,2, \ldots$

THEOREM 5.1. $\Phi(X)=\bigcup_{a=1}^{\infty} M_{a}(X), \quad \Phi(Y)=\bigcap_{a=1}^{\infty} \tilde{M}_{a}(Y)$.

This theorem is a simple consequence of Theorem 1.2.

TheoreM 5.2. Let $X=\mathscr{M}\left(a_{n}^{\alpha}\right)$ and $Y=\mathscr{M}\left(b_{n}^{1 / a}\right)$ be power spaces of infinite type and of finite type, respectively. Then $\mathrm{d}_{a} X \neq \mathrm{d}_{a} Y$; therefore $X \neq Y$.

Proof. Suppose that

$$
\Phi(X) \supset \Phi(Y) .
$$

We shall prove that in this case $\Phi(X) \supset \Phi(Y)$.

From the definition of functions $M_{a}(X ; \varepsilon)$ and $\tilde{M}_{a}(Y ; \varepsilon)$ it follows that

(54) $\quad \ldots \dot{<} M_{2}(X ; \varepsilon) \ddot{<} M_{1}(X ; \varepsilon) ; \quad \tilde{M}_{1}(Y ; \varepsilon) \dot{<} \tilde{M}_{2}(Y ; \varepsilon) \dot{<} \ldots$,

whence, by Theorem 5.1 and by formula (53), we have

$$
\tilde{M}_{a}(Y ; \varepsilon) \dot{<} M_{a}(X ; \varepsilon) \quad(\alpha=1,2, \ldots) .
$$


Inequalities (54) and (55) imply the existence of a decreasing sequence $\left(\varepsilon_{k}\right)$, with $\lim _{k \rightarrow \infty} \varepsilon_{k}=0$, such that

$$
M_{1}(X ; \varepsilon)>M_{2}(X ; \varepsilon)>\ldots>M_{k}(X ; \varepsilon)>\tilde{M}_{k}(Y ; \varepsilon)>\tilde{M}_{k-1}(Y ; \varepsilon)>
$$$$
\ldots>\tilde{M}_{1}(\bar{Y} ; \varepsilon) \text { for } \varepsilon<\varepsilon_{k} \text {. }
$$

Put

$$
\varphi(\varepsilon)=\left\{\begin{array}{cll}
1 & \text { for } & \varepsilon \geqslant \varepsilon_{1}, \\
\frac{1}{2}\left(M_{k}(X ; \varepsilon)+\tilde{M}_{k}(\bar{Y} ; \varepsilon)\right) & \text { for } & \varepsilon_{k}>\varepsilon \geqslant \varepsilon_{k+1} .
\end{array}\right.
$$

We have

$$
M_{l_{k}}(X ; \varepsilon) \dot{>} \varphi(\varepsilon)>\tilde{M}_{k}(\bar{Y} ; \varepsilon)
$$

this means, according to Theorem 5.1, that $\Phi(X) \neq \Phi(Y)$, q. e. d.

2. LeMra 5.1. Let $X$ be a nuclear space that satisfy the following condition:

(56) $\quad \varphi \epsilon \Phi(X)$ if and only if $\varphi^{2} \epsilon \Phi(X)$.

If $X=\mathscr{M}\left(a_{n}^{a}\right)$, then $\Phi(X)=\bigcup_{\alpha}\left\{\varphi: \log \varphi(\varepsilon) / \log \frac{1}{\varepsilon} \geqslant N(\sqrt[a]{\varepsilon})\right\} ;$ if $X=\mathscr{M}\left(b_{n}^{1 / \alpha}\right)$, then $\Phi(X)=\bigcap_{a}\left\{\varphi: \log \varphi(\varepsilon) \log \frac{1}{\varepsilon} \geqslant \tilde{N}\left(\varepsilon^{\alpha}\right)\right\}$, where $N(\varepsilon)=$ $\overline{\overline{\left\{n: a_{n} \leqslant 1 / \varepsilon\right\}}}, \quad \tilde{N}(\varepsilon)=\overline{\overline{\left\{n: b_{n} \geqslant \varepsilon\right\}}}$.

Proof. $1^{\circ} X=\mathscr{M}\left(a_{n}\right)$. Theorem 1.3, for $k=1$, gives us

$$
2 N(\sqrt[\alpha]{\varepsilon}) \log \frac{1}{\varepsilon} \geqslant \log M_{a}(X ; \varepsilon) \geqslant \frac{1}{2} N(\sqrt[2 \alpha]{\varepsilon}) \log \frac{1}{\varepsilon}
$$

Let $\varphi \epsilon \Phi(X)$. Then, by $(56), \sqrt{\varphi \epsilon} \Phi(X)$ and, according to Theorem 5.1, we have

$$
\varphi \epsilon \bigcup_{a}\left\{\varphi: \frac{1}{2} \log \varphi(\varepsilon) \geqslant \frac{1}{2} N(\sqrt[a]{\varepsilon}) \log \frac{1}{\varepsilon}\right\}
$$

On the other hand, if $\varphi \in \bigcup_{a}\left\{\varphi: 2 \log \varphi(\varepsilon) \geqslant 2 N(\sqrt[a]{\varepsilon}) \log \frac{1}{\varepsilon}\right\}$, then $\varphi^{2} \epsilon \Phi(X)$ and, by $(56), \varphi \in \Phi(X)$.

$2^{0} X=M\left(b_{n}^{1 / \alpha}\right)$. The proof in this case is based on the inequality

$$
2 \tilde{N}\left(\varepsilon^{\alpha}\right) \log \frac{1}{\varepsilon} \geqslant \log \tilde{M}_{\alpha}(X ; \varepsilon) \geqslant \frac{1}{2} \tilde{N}\left(\varepsilon^{\alpha / 2}\right) \log \frac{1}{\varepsilon} .
$$

Remark 5.1. It is easily seen that in Lemma 5.1 assumption $(56)$ may be replaced by " $\psi \epsilon \bigcup_{a}\left\{\psi: \psi(\varepsilon) \geqslant N\left(\sqrt[a]{\varepsilon)}\left(\right.\right.\right.$ resp. $\left.\psi \epsilon \bigcup_{a}\left\{\psi: \psi(\varepsilon) \geqslant \tilde{N}\left(\varepsilon^{a}\right)\right\}\right)$ if and only if $2 \psi \epsilon \bigcup_{a}\left\{\psi: \psi(\varepsilon) \geqslant \tilde{N}(\sqrt[a]{\varepsilon)}\}\right.$ (resp. $\left.2 \psi \epsilon \bigcap_{a}^{a}\left\{\psi: \psi(\varepsilon) \geqslant \tilde{N}\left(\varepsilon^{a}\right)\right\}\right)$ ".

THEOREM 5.3. Let $X$ and $X_{1}$ be power spaces satisfying condition (56) and let $\mathrm{d}_{a} X=\mathrm{d}_{a} X_{1}$. Then the spaces $X$ and $X_{1}$ are isomorphic.

Proof. $1^{0} X=\mathscr{M}\left(a_{n}^{a}\right), X_{1}=\mathscr{M}\left(c_{n}^{\alpha}\right)$. Suppose that conditions (51) and (52) are satisfied. Let $N(\varepsilon)$ and $N_{1}(\varepsilon)$ be functions defined in Lemma 5.1, for the spaces $X$ and $X_{1}$, respectively. By Lemma 5.1, we have

$$
\bigcup_{\alpha}\{\psi: \psi(\varepsilon) \geqslant N(\sqrt[a]{\varepsilon})\}=\bigcup_{a}\left\{\psi: \psi(\varepsilon) \geqslant N_{1}(\sqrt[\alpha]{\varepsilon})\right\},
$$

whence $N(\varepsilon) \in \bigcup_{a}\left\{\psi: \psi(\varepsilon) \geqslant N_{1}(\sqrt[\alpha]{\varepsilon)})\right\}$ and $N_{1}(\varepsilon) \in \bigcup_{a}\{\psi: \varphi(\varepsilon) \geqslant N(\sqrt[a]{\varepsilon})\}$. Thus there exist positive integers $\alpha_{0}$ and $\beta_{0}$ such that $N(\varepsilon) \geqslant N_{1}(\sqrt[\mathbb{\varepsilon} \alpha]{\varepsilon})$, $N_{1}(\varepsilon) \geqslant N\left(\sqrt[\beta_{0}]{\varepsilon}\right)$. Sequences $\left(a_{n}\right),\left(c_{n}\right)$ being non-decreasing, this implies that, for sufficiently large $n, a_{n} \leqslant c_{n}^{a_{0}}$ and $a_{n} \leqslant c_{n}^{\beta_{0}}$, i. e. the identical mapping $T\left(\xi_{n}\right)=\left(\xi_{n}\right)$ is the required isomorphism from $X$ onto $X_{1}$.

$2^{\circ} X=\mathscr{M}\left(b_{n}^{1 / \alpha}\right), X_{1}=\mathscr{M}\left(d_{n}^{1 / a}\right)$. Let $\left(b_{n}\right)$ and $\left(d_{n}\right)$ satisfy conditions (51) and (52). Denote by $\tilde{N}(\varepsilon)$ and $\tilde{N}_{1}(\varepsilon)$ the function defined in Lemma 5.1 for the spaces $X$ and $X_{1}$, respectively. By Lemma 5.1

$$
\bigcap_{a}\left\{\psi: \psi(\varepsilon) \geqslant \tilde{N}\left(\varepsilon^{a}\right)\right\}=\bigcap_{a}\left\{\psi: \psi(\varepsilon) \geqslant \tilde{N}_{1}\left(\varepsilon^{a}\right)\right\} .
$$

We shall show that there exists a positive integer $a_{0}$ such that $\tilde{N}\left(\varepsilon^{a_{0}}\right) \geqslant \tilde{N}_{1}(\varepsilon)$.

In fact, if the last inequality held for no $\alpha$, then there would exist a decreasing sequence $\left(\varepsilon_{a}\right)$, with $\lim \varepsilon_{a}=0$, such that $\tilde{N}\left(\varepsilon_{a}^{a}\right)<\tilde{N}_{1}\left(\varepsilon_{a}\right)$. Let

$$
\psi(\varepsilon)= \begin{cases}1 & \text { for } \quad \varepsilon>\varepsilon_{1}, \\ \tilde{N}\left(\varepsilon^{a}\right) & \text { for } \quad \varepsilon_{\alpha} \geqslant \varepsilon>\varepsilon_{\alpha+1} .\end{cases}
$$

Then $\psi \epsilon \bigcap_{a}\left\{\psi: \psi(\varepsilon) \geqslant \tilde{N}\left(\varepsilon^{a}\right)\right\}$ and $\psi \xi \bigcap_{a}\left\{\psi: \psi(\varepsilon) \geqslant \tilde{N}_{1}(\varepsilon)\right\}$, which contradicts formula $(57)$.

Now $\tilde{N}\left(\varepsilon_{n}^{\alpha_{0}}\right) \geqslant \tilde{N}_{1}(\varepsilon)$ implies that, for sufficiently large $n, d_{n} \geqslant b_{n}^{\alpha_{0}}$. The assumption of the theorem being symmetric with respect to $X$ and $X_{1}$, we have also $b_{n} \geqslant d_{n}^{\beta_{0}}$ for sufficiently large $n$. Hence the mapping $T\left(\xi_{n}\right)=\left(\xi_{n}\right)$ is the required isomorphism from $X$ onto $X_{1}$, q. e. d.

Since nuclear Köthe spaces of type $\mathscr{M}\left(a_{n}^{\alpha}\right), \mathscr{M}\left(b_{n}^{1 / \alpha}\right)$ are isomorphic to power spaces, we have 
COROLLARY 5.1. If $X=\mathscr{M}\left(a_{n}^{\alpha}\right), X_{1}=\mathscr{M}\left(c_{n}^{\alpha}\right), Y=M\left(b_{n}^{1 / \alpha}\right), Y_{1}=$ $=M\left(d_{n}^{1 / a}\right)$ are nuclear Köthe spaces satisfying condition (56) and $\mathrm{d}_{a} X=$ $=\mathrm{d}_{a} X_{1}, \mathrm{~d}_{a} Y=\mathrm{d}_{a} Y_{1}$, then $X=X_{1}, Y=Y_{1}$. Hence

Spaces considered in $\S 2.3$, sections 1 and 2 , satisfy condition (56).

Corollary 5.2. Let

$$
X=\mathscr{M}\left(\exp \left(\alpha\left(n_{1}^{q_{1}}+\ldots+n_{r}^{q_{r}}\right)-\frac{1}{\alpha}\left(n_{r+1}^{q_{r+1}}+\ldots+n_{r}^{q_{r}}\right)\right)\right)
$$

with $q_{i}>0$, and let $q=\left(1 / q_{1}+\ldots+1 / q_{k}\right)^{-1}$. Then if $r=0$, then $X \approx M\left(\exp -n^{q} / a\right) ;$ if $r=k$, then $X \approx \mathscr{M}\left(\exp \alpha n^{q}\right)$.

COROLlaRY 5.3. Let $X=\mathscr{M}\left(\left(n_{1}^{n_{1}} \ldots n_{r}^{n_{r}}\right)^{\alpha} \cdot\left(n_{r+1}^{n_{r+1}} \ldots n_{k}^{n_{k}}\right)^{-1 / \alpha}\right)$. If $r=0$, then $X=\mathscr{M}\left(n^{-\sqrt[k]{n} / a}\right)$; if $r=k$, then $X \approx \mathscr{M}\left(n^{a \sqrt{k}}{ }^{\sqrt[k]{n}}\right)$.

To prove these two corollaries we apply Theorems 4.2 and 4.3 for an arbitrary $k$ and for $k=1$ and verify that suitable spaces have an equal approximative dimension.

3. In general, Köthe spaces with the same approximative dimension need not be isomorphic.

Example 5.1. Let $X=\mathscr{M}(\exp \alpha n), \quad Y=(X \times X \times \ldots)_{s}$. Then $\mathrm{d}_{a} X=\mathrm{d}_{a} Y$, but $X \neq Y$.

The quality of approximative dimensions follows from Theorem 1.5. $X \neq Y$, because there exist continuous (homogeneous) norms defined on $X$; in the space $s$ (of all numerical sequences), which is isomorphically contained in $Y$, no continuous pseudonorm is a norm.

The next example will concern the spaces in which there are continuous norms.

Example 5.2. Let $X=\mathscr{H}\left(C_{0}\right), \quad Y=\mathscr{H}\left(C_{0}\right) \times \mathscr{H}(C)$. Then $\mathrm{d}_{a} X=$ $=\mathrm{d}_{a} Y$, but $X \neq Y$.

The equality of $\mathrm{d}_{a}$ follows from the inclusions $X \approx X \times X \supset Y \supset X$ (see Theorems 2.2 and 2.4).

To prove that $X$ and $Y$ are not isomorphic, we shall apply the following result of Dragilev [7]:

Every basis $\left(e_{n}\right)$ of the space $\mathscr{H}\left(C_{0}\right)$ is o-equivalent to the basis $\left(z^{n}\right)$, i. e. there exist a sequence $\left(\tau_{n}\right)$ of positive numbers and a permutation $\left(p_{n}\right)$ of indices such that the series $\sum_{n} t_{n}\left(\tau_{n} e_{p_{n}}\right)$ converges if and only if the series $\sum t_{n} z_{n}$ converges.

If $X \approx Y$ then the space $\mathscr{H}\left(C_{0}\right)$ would be isomorphic to the space $\mathscr{M}\left(c_{a n}\right)$, where $c_{a n}=\exp \alpha n$ for $n=2 m-1, c_{\alpha n}=\exp -n / \alpha$ for $n=2 m$ Now according to the theorem of Dragilev there would exist an increa- sing sequence $\left(k_{n}\right)$ of non-negative integers such that $\mathscr{M}(\exp \alpha, n) \approx$ $\approx \mathscr{M}\left(\exp -k_{n} / \alpha\right)$, which contradicts Theorem 5.2.

\section{General remarks. Let}

$$
\Psi(X)=\left\{\psi(\varepsilon)=\log \varphi(\varepsilon) / \log \frac{1}{\varepsilon}: \varphi \epsilon \Phi(X)\right\}
$$

Condition (56) formulated in terms of $\Psi(X)$ has a form

$\left(56^{\prime}\right) \quad \psi \epsilon \Psi(X)$ if and only if $2 \psi \epsilon \Psi(X)$.

It is easy to prove the following

THEOREM 5.4. Let $X$ and $Y$ be power spaces satisfying condition (56'). Then $\Psi(X \times Y)=\left\{\psi=\psi_{1}+\psi_{2}: \psi_{1} \in \Psi(X), \quad \psi_{2} \in \Psi(Y)\right\}, \quad \Psi(X \hat{\otimes} Y)=$ $\left\{\psi=\psi_{1} \cdot \psi_{2}: \psi_{1} \in \Psi(X), \psi_{2} \in \Psi(Y)\right\}$.

All the spaces considered in $\S 4$ are tensor products of $k$ copies of single Köthe spaces; the results of $\S 4$ are illustrations of Theorem of $\S 4$.

The theorem of Dragilev cited in section 3 has been generalized by Mitiagin [20] in the following way:

In an arbitrary power space all bases are e-equivalent.

Applying this theorem, by the same consideration as in Example 5.2 we obtain

COROLLARY 5.4. If $X$ and $Y$ are power spaces of finite and of infinite type, respectively, then $X \times Y \neq X, X \times Y \neq Y ; X \hat{\otimes} Y \neq X$, $X \hat{\otimes} Y \neq Y$.

In particular we have

Example 3. Let $X_{i}=\mathscr{M}\left(\exp \left(\alpha\left(n_{1}^{q_{1}}+\ldots+n_{r_{i}}^{q_{r_{i}}}\right)-\frac{1}{\alpha}\left(n_{r_{i}+1}^{q_{r_{i}+1}}+\ldots+\right.\right.\right.$

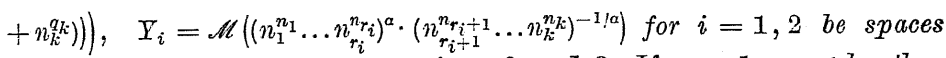
of type considered in $\S 3,4$, sections 2 and 3 . If $r_{1}=1, r_{2}<k$, then $\mathrm{d}_{a} X_{1}=\mathrm{d}_{a} X_{2}, \quad \mathrm{~d}_{a} Y_{1}=\mathrm{d}_{a} Y_{2}$. If $r_{1}=1, r_{2}>1$ or $r_{1}<k, r_{2}=k$, then $X_{1} \neq X_{2}$ and $Y_{1} \neq Y_{2}$.

We do not know whether $\left(56^{\prime}\right)$ is true in the case where $1<r_{1}<$ $<r_{2}<k$; in particular we do not know whether $H\left(C_{0} \times C^{2}\right) \neq H\left(C_{0}^{2} \times C\right)$ ? This is obviously connected with the following.

Conjecture. Let $X_{1}$ and $Y_{1}$ be power spaces of infinite type, let $X_{2}$ and $Y_{2}$ be power spaces of finite type and let $X_{1}, Y_{1}, X_{2}, Y_{2}$ satisfy condition (56). If $X_{1} \neq Y_{1}$ or $X_{2} \neq Y_{2}$, then $X_{1} \hat{\otimes} X_{2} \neq Y_{1} \hat{\otimes} Y_{2}$ (cf. Problem 2.1). 


\section{\$6. Estimation of Taylor coefficients}

Let $\boldsymbol{n}=\left(n_{1}, \ldots, n_{k}\right)$. We shall write $|\boldsymbol{n}|=n_{1}+\ldots+n_{k}$.

THEOREM 6.1. Let $q>0 . \quad\left(\xi_{n}\right) \in \mathscr{M}\left(\exp \alpha|\boldsymbol{n}|^{q}\right)$ if and only if $|\boldsymbol{n}|^{q}$

$\lim _{\mid \boldsymbol{n} i \rightarrow \infty} \sqrt[|\boldsymbol{n}|^{q}]{\left|\xi_{\boldsymbol{n}}\right|}=0 . \quad\left(\xi_{\boldsymbol{n}}\right) \in \mathscr{M}\left(\exp -|\boldsymbol{n}|^{q} / \alpha\right)$ if and only if $\lim _{|\boldsymbol{n}| \rightarrow \infty} \sqrt[|\boldsymbol{n}|^{q}]{\left|\xi_{\boldsymbol{n}}\right|} \leqslant 1$.

Proof. $1^{\circ}$ The following conditions are equivalent:

$2^{\circ}$ The following conditions are equivalent:

q. e. d.

$$
\begin{gathered}
\left(\xi_{n}\right) \in \mathscr{M}\left(\exp \left(-|\boldsymbol{n}|^{q} / \alpha\right)\right), \\
\left.|\boldsymbol{n}|^{q} / \alpha\right)=-\infty \quad \text { for } \\
\varlimsup_{|\boldsymbol{n}| \rightarrow \infty} \frac{\log \xi_{\boldsymbol{n}}}{|\boldsymbol{n}|^{\boldsymbol{\alpha}}}=0, \\
\varlimsup_{|\boldsymbol{n}| \rightarrow \infty}\left|\xi_{\boldsymbol{n}}\right|^{1 /|\boldsymbol{n}|^{q}} \leqslant 1,
\end{gathered}
$$$$
\lim _{|\boldsymbol{n}| \rightarrow \infty}\left(\log \left|\xi_{\boldsymbol{n}}\right|-|\boldsymbol{n}|^{q} / \alpha\right)=-\infty \quad \text { for } \quad \alpha=1,2, \ldots,
$$

Applying Theorems $3.1,3.4,3.6$ we obtain

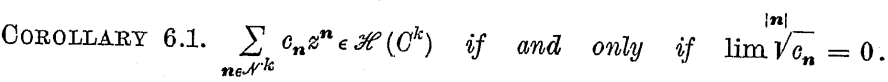
$\sum_{\boldsymbol{n} \in \mathcal{W}^{k}} e_{\boldsymbol{n}} z^{n} \in \mathscr{H}\left(C_{0}^{k}\right)$ if and only if $\lim \sqrt[|\boldsymbol{n}|]{e_{\boldsymbol{n}}} \leqslant 1 \quad$ (where $\boldsymbol{n}=\left(n_{1}, \ldots, n_{k}\right)$, $\left.z^{\boldsymbol{n}}=z_{1}^{n_{1}} \ldots z_{k}^{n_{k}}\right)$.

CoRoliari 6.2. Let $\mu(\varepsilon, z)=\exp \left(-\left(\tau_{1}+\varepsilon\right)\left|z_{1}\right|^{p_{1}}-\ldots-\left(\tau_{k}+\varepsilon\right)\left|z_{k}\right|^{p_{k}}\right)$ with $\tau_{i} \geqslant 0, p_{i}>0$. If $\tau_{1}=\ldots=\tau_{k}=0$, then $\sum_{n \in \mathcal{N}^{k}} c_{\boldsymbol{n}} z^{\boldsymbol{n}} \epsilon \mathscr{H}_{\mu}$ if and only

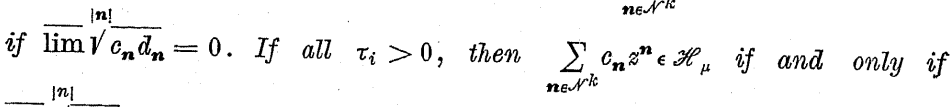
$\lim \sqrt[|n|]{c_{n} d_{n}} \leqslant 1$, where $d_{n}$ are given by formula (35).
Corollary 6.3. Let $\mu(\varepsilon, z)=\exp \left(-\varepsilon(\log 1 / z)^{-s}\right)$, with $s>0$. Then $\sum_{n=0}^{\infty} c_{n} z^{n} \in \mathscr{H}_{\mu}\left(C_{0}\right)$ if and onty if $\varlimsup_{10} \sqrt[n]{c_{n}} \leqslant 1$, where $q=s /(s+1)$.

\section{References}

[1] Л. А. Ай вен берг, Пространства функиий аналитических в $(p, q)$. -круговых областля, ДАН 136 (1961). p. 521-524.

[2] Л. А. Айвенберг и Б. С. Митягин, Пространства функиий аналитических в кратно-круговых област.ях, Спб. Матем. Ж. I (1960), p. 153-169.

[3] S. Banach, Théorie des opérations linéaires, Warszawa-Lwów 1932.

[4] М. Бессага иг A. Пелчпнски, О в.ложении ядерных пространств в пространство всех бесконечно дифференцируемых функций на пря.мой, ДАН 134 (1960), p. $745-748$.

[5] C. Bessaga, A. Pełczyński and S. Rolewicz, Approximative dimension of linear topological spaces and some its applications, Reports of Conference of Functional Analysis, Warszawa 1960.

[6] N. Bourbaki, Éléments de Mathématique, Livre V. Espaces vectoriels topologiques.

[7] M. М. Др агилев, Каноническал форма базиса пространства аналитических функций, Усп. мат. наук 15 (1960), 2 (92), p. 181-188.

[8] A. Dynin and B. Mitiagin, Criterion for nuclearity in terms of approximative dimension, Bull. Acad. Pol. Sci., Cl. III. 8 (1960), p. 535̈-540.

[9] В. Д. Ерохин. Об асимптотике и в-энтропии аналитических функиий, ДАН 120 (1958), p. 949-952.

[10] Б. А. ФУкс, Теория аналитических функиий мнсеих комплексных переменных, Москва-Ленинград 1948.

[11] A. Grothendieck, Produits tensoriels topologiques et espaces nucléaires, Mem. Amer. Math. Soc. 16 (1955).

[12] - Sur certains espaces de fonctions holomorphes, I, J. reine u. angew. Math. 192 (1953), pp. 35-64, 77-95.

[13] Г. М. Голувпн. Геометрическал теория. функций помплепсного переменного, Москва-Ленинград 1952.

[14] А. Н. Колмогоров, О линейной размерности топологических векторных пространст, ДАН 120 (1958), р. 239-241.

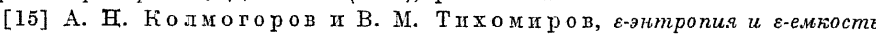
множеств в фуннкииона.льных пространствах, Усп. Мат. наук 14 (1959), 2 (80), p. $3-86$.

[16] G. Köthe, Die Stufenräume, eine einfache Klasse linear vollkommener Räume, Math. Zeitschrift 51 (1949), p. $317-345$.

[17] - Dualität in Funktionentheorie, J. reine u. angew. Math. 191 (1953), p. $30-49$.

[18] S. Mazur et W. Orlicz, Sur les espaces linéaires métriques: I, Studia Math. 10 (1948), p. 184-208; II, ibidem 13 (1953), p. 137-179.

[19] Б. С. Митя яг ин, Связь межәу в-әнтропией, скоростью аппроксимации и ядерностью компакта в линейном пространстве, ДАН 134 (1960), 765-768.

[20] - Ядерные икалы Pисса, ibidem 137 (1961), p. 519-522.

[21] A. Pelczyński, On the approximation of S-spaces by finite dimensional spaces, Bull. Acad. Pol. Sei., Cl. III. 5 (1957), p. 879-881. 
[22] S. Rolewicz, Remarks on linear metric Montel spaces, ibidem III. 7 (1959), p. $195-197$.

[23] - On isomorphic representation of spaces of holomorphic functions by ma. rix spaces $M\left(a_{m}, n\right)$, Reports of the Conference on Functional Analysis, Warszawa 1960 .

[24] С. Р олев и ч, Об изоморфизме и аппроксимативной размерности пространств голоморфных функиий, ДА耳 133 (1960), p. 31-33.

Reģu par la Rédaction le 8.12. 1960
Bases, lacunary sequences and complemented subspaces in the spaces $L_{p}$

by

M. I. KADEC (Kharkov) and A. PEECZYŃSKI (Warszawa)

In this paper we investigate the isomorphic structure (invariants of linear homeomorphisms) of subspaces of the space $L_{p}(1 \leqslant p<+\infty)$. We consider especially the properties of basic sequences (bases in subspaces), as well as the properties of subspaces complemented in $L_{p}$. These properties are connected with classical problems concerning lacunary series. We explain them in a more detailed way.

Let $p>2$ and let $\left(\varphi_{n}\right)$ be an orthonormal system. Then

$$
\left(\int_{0}^{1}\left|\sum_{i=1}^{n} t_{i} \varphi_{i}(t)\right|^{p} d t\right)^{1 / p} \geqslant\left(\int_{0}^{1}\left|\sum_{i=1}^{n} t_{i} \varphi_{i}(t)\right|^{2} d t\right)^{1 / 2}=\left(\sum_{i=1}^{n}\left|t_{i}\right|^{2}\right)^{1 / 2}
$$

for any scalars $t_{1}, t_{2}, \ldots, t_{n}(n=1,2, \ldots)$.

An orthonormal system is said to be $p$-lacunary iff $\left({ }^{1}\right)$ the converse inequality

$$
\left(\int_{0}^{1}\left|\sum_{i=1}^{n} t_{i} \varphi_{i}(t)\right|^{p} d t\right)^{1 / p} \leqslant C\left(\sum_{i=1}^{n}\left|t_{i}\right|^{2}\right)^{1 / 2}
$$

holds for some $C$ depending only on $\left(\varphi_{n}\right)$ and for any $t_{1}, t_{2}, \ldots, t_{n}$ $(n=1,2, \ldots)$.

In the language of the functional analysis this means that there is an isomorphism (linear homeomorphism) of Hilbert space $l_{2}$ onto the closed linear manifold in $L_{p}$ spanned on the functions $\varphi_{n}$. Under this isomorphism the unit vectors in $l_{2}$ correspond the functions $\varphi_{n}$, i.e. the basic sequence $\left(\varphi_{n}\right)$ is equivalent to the unit vector basis in $l_{2}$ (see the definition in section 1). Moreover, the operator $T: x \rightarrow\left(\int_{0}^{1} x(t) \varphi_{n}(t) d t\right)$ is a projection of $L_{p}$ onto this manifold.

(1) We write "iff" instead of "if and only if". 